\title{
LE PROVE INVALSI E LA RIFLESSIONE SULLA LINGUA NELLA SCUOLA PRIMARIA: UN'INDAGINE SULLA PERCEZIONE DEGLI INSEGNANTI
}

\author{
Manuela Roccia ${ }^{1}$
}

\section{INTRODUZIONE}

Questo articolo nasce da un'indagine condotta nel mese di febbraio 2021 con l'obiettivo di esplorare gli atteggiamenti, le conoscenze e le esperienze professionali dei docenti di scuola primaria del territorio cuneese e della città di Torino rispetto alle Prove Invalsi. Contemporaneamente, l'intento è quello di verificare quanto queste ultime si riflettano o meno nella scelta di adottare un particolare modello di grammatica, se sia presente una pratica didattica finalizzata al miglioramento e allo sviluppo della competenza riflessiva degli allievi, con un'attenzione specifica nei confronti della sezione del Quadro di Riferimento delle prove Invalsi di italiano ${ }^{2}$ dedicata alla Riflessione sulla lingua.

La ricerca si sofferma sulle caratteristiche degli approcci didattici utilizzati dai docenti in questo ambito, ponendo in luce le tipologie di attività proposte per stimolare la riflessione sulla lingua e la quantità di spazio dedicato a ciascun contenuto grammaticale previsto dalle Indicażioni Naz̧ionali del curricolo per l'infanz̧ia e la scuola del primo ciclo del $2012^{3}$ e agli elementi illustrati nel Quadro di Riferimento delle prove Invalsi di italiano del 2018.

L'articolo si suddivide in due sezioni: nella prima parte si illustrano i legami esistenti tra la teoria disciplinare e la prospettiva didattica, proseguendo con l'analisi dei nessi presenti tra la didattica della grammatica e le prove Invalsi, infine si individuano le possibili affinità elettive tra i documenti ministeriali del 2012 ed il Quadro di Riferimento del 2018.

Nella seconda parte si propone una fotografia della realtà emersa dallo studio delle evidenze rilevate tramite il questionario compilato dagli insegnanti. In particolare, la trattazione si occupa di descrivere cinque macrotemi emersi:

1) l'organizzazione di un istituto scolastico come sistema complesso;

2) la percezione rispetto all'inclusione dei bisogni educativi;

3) l'utilizzo delle prove Invalsi e degli esiti relativi nella progettazione didattica d'istituto e curricolare in un'ottica di miglioramento;

4) le finalità e gli obiettivi generali dell'Invalsi e, in particolare, per la riflessione linguistica;

5) le scelte metodologico didattiche utilizzate nell'insegnamento della riflessione linguistica ed i contenuti proposti nel percorso didattico.

L'ultima parte della disamina si concentra sugli ambiti e sui contenuti della riflessione linguistica, con un'attenzione dedicata ai risultati ricavati dallo studio ed una loro analisi in chiave sia disciplinare sia didattica.

\footnotetext{
${ }^{1}$ Università degli Studi di Torino.

2 https://invalsi-areaprove.cineca.it/docs/file/QdR_ITALIANO.pdf.

${ }^{3}$ http://www.indicazioninazionali.it/wp-content/uploads/2018/08/Indicazioni_Annali_Definitivo.pdf.
} 
(C) Italiano LinguaDue 2. 2021. M. Roccia, Le prove Invalsi e la riflessione sulla lingua nella scuola primaria: un'indagine sulla percezione degli insegnanti

\section{LA GRAMMATICA E LA RIFLESSIONE LINGUISTICA: TRA TEORIA E DIDATTICA}

\subsection{I legami tra la disciplina linguistica e la prospettiva didattica}

Nell'analizzare e descrivere la complessità dell'insegnamento apprendimento della grammatica, De Mauro individua ed illustra la distinzione tra grammatica vissuta e metalinguistica. Nel primo caso, si tratta di grammaticalità, che è insita nell'uso stesso di qualsiasi lingua, comprendendo «l'intero repertorio di morfi e regole di combinazione dei morfi grammaticali» (De Mauro, 2011: 21). La grammaticalità svolge compiti differenti: infatti, nelle realizzazioni linguistiche possiede più funzioni, quali quella semantica, sistemica, sintattica e pragmatica. Appare come un elemento irrinunciabile del linguaggio, permettendone il funzionamento.

Può essere non insegnata, non lo è stata per centinaia e migliaia di anni per la stragrande maggioranza di lingue. Ma può essere oggetto di osservazione e stimolazione di apprendimenti [...] (ibidem).

Secondo De Mauro, si rivelerebbe proficuo usare l'esplorazione della grammaticalità come potenzialità del parlato e dello scritto, permettendo di percepire le possibilità offerte dai mezzi linguistici. Non tutti i fenomeni linguistici sono ascrivibili all'interno del sistema standard, anzi, molti sono gli esempi che dimostrano come

nello stesso standard si registrano oscillazioni e conseguenti non illegittime incertezze d'uso [...]. Quest'ordine di fenomeni, se in sede teorica spinge ad abbandonare l'idea di una lingua come un monolito in cui tutto si tiene e si tiene in un solo modo e a intendere che nell'uso di una lingua occorre postulare la natura anche "locale" e non sistemica di molti fenomeni di produzione e comprensione, nemmeno può restare senza conseguenze nell'osservazione dei processi di apprendimento di una lingua, anche materna, e nella progettazione di eventuali interventi didattici di sostegno alla progressiva espansione delle capacità di dominio dell'apparato grammaticale nell'uso effettivo, nel vissuto di tale uso (De Mauro, 2009: 21).

Per tali ragioni, non è sufficiente esplorare la lingua esclusivamente con la grammatica implicita (De Mauro, 2009: 17) tanto che, nel processo di apprendimento, appare essenziale accompagnare progressivamente gli allievi ad una riflessione in cui siano presenti gli strumenti propri della grammatica esplicita, attraverso proposte didattiche che tengano conto del percorso ideale di complessità sia linguistica sia cognitiva, quindi senza cadere in trappole legate ad una visione adulta del sistema linguistico. Secondo De Mauro si rivelerebbe appropriato l'ingresso graduale di una «nomenclatura metalinguistica minima, come nome, verbo, aggettivo, numero, tempo, persona, modo, pronome, preposizione, forse predicazione, il cui uso appropriato sia garantito da esemplificazioni concrete più che da definizioni generali, difficilmente esemplificabili se devono avere qualche rigore» (De Mauro, 2009: 21).

Contemporaneamente, si manifesta come necessità ineludibile il tener conto dell'attività di riflessione spontanea tipica del bambino, in particolare ciò che Hopper definisce emergent grammar, grammatica emergente, ossia il processo di acquisizione spontanea che il bambino compie sia attraverso l'interazione con il mondo circostante sia con l'attività epilinguistica (Ferreri, 2009: 27).

La medesima prospettiva disciplinare è presente nei documenti per la scuola, in particolare nelle Indicazioni Nazionali per il curricolo della scuola dell'infanzia e del primo ciclo 
d'Istruzione (2012), in cui si richiamano in modo esplicito tali processi, che si avviano con l'interazione orale nella scuola dell'infanzia e si ampliano nella scuola primaria, in sinergia con lo sviluppo cognitivo dell'apprendente.

Ogni persona, fin dall'infanzia, possiede una grammatica implicita, che le permette di formulare frasi ben formate pur senza conoscere concetti quali quelli di verbo, soggetto, ecc. Questa "grammatica implicita" si amplia e si rafforza negli anni attraverso l'uso della lingua, che permette di giungere a forme "corrette" (in italiano standard) e di realizzare enunciati in diverse varietà linguistiche e in diverse lingue. Inoltre, sin dai primi anni di scolarità, $\mathrm{i}$ bambini hanno una naturale predisposizione a riflettere sulla lingua. $\grave{E}$ su queste attitudini che l'insegnante si può basare per condurre gradualmente l'allievo verso forme di "grammatica esplicita".

(Indicażioni Nazionali per il curricolo della scuola dellinfanzia e del primo ciclo d'Istruzione, 2012: 30)

La riflessione sulla lingua nella scuola primaria non può dunque non tener conto della grammatica emergente, che si sviluppa nell'ambiente sociale, e del fondamentale ruolo che, nella pratica didattica, svolge l'interazione dialogica. In essa assume particolare rilievo l'abilità di comprensione all'interno dello scambio comunicativo, che diventa a poco a poco più raffinata, grazie alla continua e costante pratica di negoziazione di significati e di riparazione autodiretta ed eterodiretta. All'interno di tale pratica si costruisce l'acquisizione spontanea della lingua e delle costruzioni grammaticali più ricorrenti, permettendone una graduale stabilizzazione verso livelli di lingua progressivamente vicini allo standard.

Altrettanto importante è il richiamo delle Indicaz̧ioni Naz̧ionali alla grammatica implicita, intesa come la propensione naturale dell'uomo ad una riflessione inconscia e non consapevole sui fatti linguistici, definita come epilinguistica inconscia che, attraverso una rielaborazione costante dei fatti linguistici a cui si è esposti, consente di appropriarsi della lingua in modo naturale (Ferreri, 2009: 28). Esiste, infine, un ulteriore livello di riflessione, ossia quello dichiarato e cosciente di metariflessione, che, in ambito scolastico, va sviluppato gradualmente attraverso proposte didattiche che tengano conto sia dell'età degli apprendenti, quindi del loro livello cognitivo, sia della naturale propensione iniziale del bambino ad operare logicamente con lo strumento linguistico.

Tenendo presente tale prospettiva teorica, si rivela fruttuoso esaminare come sia affrontata la riflessione grammaticale nel Quadro di Riferimento delle prove Invalsi di italiano, documento che illustra i paradigmi teorici di riferimento da cui traggono ispirazione le stesse consegne contenute nei test somministrati annualmente nelle scuola italiana dall'ente di ricerca. Anche nel Quadro di Riferimento delle prove Invalsi di italiano è presente una descrizione della grammatica intesa sia come insieme di regole che sottendono e permettono il funzionamento della lingua sia in termini di grammatica implicita ed esplicita; nel Quadro di Riferimento la competenza grammaticale evidenzia la presenza di:

- una "grammatica implicita", a cui tutti i parlanti di una lingua materna fanno riferimento non solo per produrre frasi ben formate, ma anche per comprenderle;

- una "grammatica esplicita" che lo studente acquisisce gradualmente nei diversi anni di scuola, imparando a classificare e a nominare gli elementi costitutivi del sistema linguistico e a formalizzare" regole" sia morfologiche sia sintattiche di funzionamento del sistema.

Se la competenza grammaticale implicita è "naturale", essa tuttavia si rafforza e si raffina negli anni attraverso un uso a mano a mano più ampio, più 
specifico e più controllato delle diverse varietà linguistiche, certamente possibile anche al di fuori della scuola, ma in buona parte appreso grazie alla scolarizzazione.

(Quadro di Riferimento delle prove Invalsi di Italiano, 2018: 6)

L'approccio teorico sopra descritto si unisce idealmente anche alle richieste contenute nello stesso Quadro di Riferimento delle prove Invalsi di italiano, in cui si propone di privilegiare lo sviluppo di una sempre maggiore e più precisa, anche linguisticamente, analisi funzionale e formale di un oggetto complesso, la lingua, e il suo corretto utilizzo in antitesi ad «una classificazione astratta e fine a sé stessa» (Quadro di Riferimento delle prove Invalsi di Italiano, 2018: 4).

Già nelle Dieci Tesi per l'educazione linguistica democratica proposte da Tullio de Mauro e fatte proprie dal Giscel nel 1975, si esprimeva l'idea che la bussola che deve guidare l'attività dell'insegnante è rappresentata dal senso generato dalla «funzionalità comunicativa ${ }^{4} »$ : ossia quell'arduo compito di avviare la riflessione sui testi, attraverso i testi stessi, per poi tornarci con maggiore consapevolezza, individuandone le caratteristiche. Il fine ultimo è quello di condurre l'allievo a sviluppare le sue capacità di comprensione e di produzione di testi adeguati alle differenti situazioni comunicative.

Su questa stessa linea, le Indicażioni Nazionali del 2012 raccomandano che la riflessione sulla lingua si basi, con un'attenzione di tipo scientifico, sull'osservazione dei diversi fenomeni linguistici alla ricerca di relazioni, di simmetrie, di regolarità e varietà interne alla lingua:

[...] il ruolo probabilmente più significativo della riflessione sulla lingua è quello metacognitivo: la riflessione concorre infatti a sviluppare le capacità di categorizzare, di connettere, di analizzare, di indurre e dedurre, utilizzando di fatto un metodo scientifico.

(Quadro di Riferimento delle prove Invalsi di Italiano, 2018: 6, 7)

Una proposta didattica finalizzata all'acquisizione di una competenza linguistica e comunicativa prevede un teorico ed auspicato passaggio da una conoscenza grammaticale implicita ad una dichiarativa e, infine, ad una conoscenza procedurale (Andorno, 2009), affinché gli elementi prima impliciti si trasformino in espliciti e vengano interiorizzati, risultando utili, funzionali e reali nella comprensione e produzione testuale. Sarà utile adottare, tuttavia, una visione lucida e pragmatica, tenendo conto dei possibili limiti di tale prospettiva, non considerandola ingenuamente come fine ultimo e diretto, bensì come un'ulteriore possibilità offerta agli apprendenti, e ricordando, in ogni caso, che

Pensare che lo studio riflesso di una regola grammaticale ne agevoli il rispetto effettivo è, più o meno, come pensare che chi meglio conosce l'anatomia delle gambe corre più svelto, chi sa più l'ottica vede più lontano ecc.

(Dieci Tesi Giscel, tesi VII, D. b)

e che appare più fruttuoso

[...] esplorare in quali circostanze, in quali età, in quali ambiti la riflessione sulle caratteristiche formali di una lingua possa tradursi in un miglioramento delle capacità di uso della lingua stessa (Lo Duca, 2018: 34)

${ }^{4}$ Dieci Tesi Giscel, Tesi VIII, 10: https://giscel.it/dieci-tesi-per-leducazione-linguistica-democratica/. 
Inoltre, in una proposta didattica di riflessione sulla e con la lingua, sarà necessario anche tenere presente l'insita dualità che contraddistingue i sistemi che permettono la costruzione di elaborati linguistici, ossia l'aspetto grammaticale della frase e la costruzione del testo, inteso come organizzazione delle frasi tra loro in una visione sovrafrasale: infatti, entrambi questi sistemi sono governati da regole e principi propri e rappresentano un insieme di costituenti che "presiedono alla costruzione del discorso, dialogando ed influenzandosi vicendevolmente» (Ferrari, 2014: 42, 43).

\subsection{I legami tra la didattica della grammatica e le prove Invalsi}

Tenendo conto dei processi che sottostanno all'acquisizione e all'apprendimento della lingua come oggetto complesso, in cui si intersecano numerosi passaggi, dalla conoscenza implicita a livelli più raffinati, fino alla comprensione e produzione di testi complessi, la riflessione grammaticale assume un ruolo più ampio e determinante nella costruzione della competenza linguistica. Non si tratta solamente di individuare e classificare le singole classi di parole e i tipi di frase, ma di condurre un'analisi composita, che permette di legare tra loro i vari costituenti della lingua, anche secondo criteri di analisi diversi che, insieme, consentono la comprensione e la creazione di testi: una sorta di continuo ritorno tra centro e periferia, tra costituenti e possibilità di significati e significazione, che risultano infiniti poiché sottostanti alla creatività linguistica.

A questo punto e sulla base di queste premesse, si rivela interessante provare a correlare elementi di educazione ed apprendimento linguistico alla loro attuazione, tramite quanto si ricava sia dalle Indicazioni Nazionali del 2012 sia dal Quadro di Riferimento dell'Invalsi.

I due documenti sottolineano, innanzitutto, l'importanza dello sviluppo della competenza linguistica nella scuola; in particolare nelle Indicazioni Nazionali, relative alla sezione Italiano, si evidenzia come

Lo sviluppo di competenze linguistiche ampie e sicure è una condizione indispensabile per la crescita della persona e per l'esercizio pieno della cittadinanza, per l'accesso critico a tutti gli ambiti culturali e per il raggiungimento del successo scolastico in ogni settore di studio.

(Indicazioni Nazionali per il curricolo della scuola dellinfanzia e del primo ciclo d'Istruzione, 2012:29)

Secondo il Quadro di Riferimento delle prove Invalsi di Italiano (2018: 3), la competenza linguistica «consiste nel possesso ben strutturato di una lingua assieme alla capacità di servirsene per i vari scopi». Le Indicaz̧ioni Nažionali analizzano in modo dettagliato le abilità insite nella competenza linguistica, considerando gli aspetti relativi all'oralità, alla lettura, alla scrittura, all'acquisizione del lessico ricettivo e produttivo, agli elementi di grammatica esplicita e alla riflessione sugli usi della lingua. Parallelamente, nel Quadro di Riferimento Invalsi si ritrovano le competenze che afferiscono alla competenza linguistica, quali l'ascolto e la produzione e l'interazione orale, la lettura e la comprensione, la scrittura, il lessico e la riflessione linguistica. Per quanto concerne il documento redatto dall'Invalsi, la valutazione delle prove risulta circoscritta a tre aree: la competenza di lettura (intesa come comprensione, l'interpretazione e la valutazione del testo scritto), le conoscenze e le competenze grammaticali e la competenza semantico lessicale ${ }^{5}$.

Pur apparentemente simili nel delineare le capacità che costituiscono la competenza linguistica, tra $\mathrm{i}$ due documenti si evince una differenza per quanto riguarda la sezione

${ }^{5}$ La competenza semantico lessicale viene tuttavia misurata solo per il grado 08 , al termine della scuola secondaria di primo grado. 
dedicata all'abilità di lettura; infatti, se nelle Indicaz̧ioni Naz̧ionali si illustrano modalità e linee di intervento per attuare un adeguato processo di insegnamento ed apprendimento della lettura, nel Quadro di Riferimento si definiscono con precisione elementi cognitivi e linguistici sottesi sia alla costruzione dell'abilità sia al suo successivo e progressivo sviluppo, con indicazioni relative alle componenti testuali e alle strategie di rielaborazione necessarie, affinché si attui una comprensione significativa dei differenti tipi di testo.

In tale processo interattivo e dinamico attuato dal lettore, le dimensioni implicate riguardano tre aree: quella pragmatico-testuale, quella lessicale e quella grammaticale. Nel Quadro di Riferimento,

La dimensione pragmatico-testuale relativa alla lettura consiste nella capacità di ricostruire, a partire dal testo, dal contesto (o "situazione") in cui esso è inserito e dalle conoscenze "enciclopediche" del lettore, l'insieme di significati che il testo veicola (il suo senso), assieme al modo in cui essi sono veicolati [...].

Per dimensione lessicale relativa alla lettura si intende specificamente la conoscenza o la capacità di ricostruire il significato di un vocabolo in un determinato contesto e di riconoscere le relazioni di significato tra vocaboli [...].

Per dimensione grammaticale relativa alla comprensione del testo si intende la capacità di ricorrere alla cosiddetta "grammatica implicita" ed eventualmente anche alla "grammatica esplicita" per capire il testo e per risolvere dubbi di comprensione.

(Quadro di Rifermento Invalsi, 2018: 4-6)

In questo quadro, l'aspetto grammaticale assume una veste originale rispetto a ciò che viene proposto nelle Indicazioni Nazionali. La riflessione grammaticale acquisisce una funzione rilevante nei processi implicati nella ricostruzione e comprensione di un testo, diventando fulcro, non solo dei legami tra le diverse parti, ma anche dei significati sottesi in relazione ai diversi modi e tempi verbali presenti, degli aspetti sintattici che permettono la comprensione e la rielaborazione del testo, ecc.

Nella nostra analisi l'attenzione si concentrerà esclusivamente sui test delle prove Invalsi dedicati alle conoscenze e competenze grammaticali, mantenendo però presenti il modello teorico e le indicazioni del Quadro di Riferimento.

\subsection{La riflessione sulla lingua proposta nelle Indicazioni Nazionali e nel Quadro di Riferimento dell'Invalsi: affinità elettive?}

Per quanto riguarda la sezione specifica relativa alla grammatica ed alla riflessione linguistica, si propone un approfondimento per enucleare i legami tra le Indicazioni Nazionali per il curricolo della scuola dell'infanzia e del primo ciclo d'istruzione del 2012 ed il Quadro di Riferimento delle prove Invalsi di italiano del 2018. Le possibili connessioni tra i due documenti permettono di evidenziare l'interdipendenza tra le linee delineate dalle Indicazioni, che rappresentano anche il materiale su cui operativamente gli istituti scolastici predispongono il comune agire attraverso il proprio curricolo verticale d'istituto e le scelte individuali dell'agire didattico del docente, tenendo conto anche della descrizione della disciplina offerta dal Quadro di Riferimento dell'Invalsi e dagli esiti ottenuti ogni anno sia a livello nazionale che di singolo istituto.

Da una lettura dei due documenti emergono i nessi esistenti tra l'approccio alla disciplina illustrato nelle Indicazioni e la competenza grammaticale descritta nel Quadro di Riferimento. Si possono individuare sei fattori comuni: 
1. l'idea della presenza di una grammatica implicita ed esplicita della lingua;

2. l'attenzione posta al livello cognitivo degli apprendenti;

3. gli oggetti di indagine linguistica da proporre;

4. un interesse specifico nei confronti del lessico;

5. una riflessione su modelli teorici sia linguistici sia didattici di riferimento;

6. l'interesse verso un approccio induttivo e di analisi funzionale dei fenomeni linguistici.

Nel paragrafo Elementi di grammatica esplicita e riflessione sugli usi della lingua delle Indicazioni nazionali del 2012 è presente un richiamo all'idea di grammatica implicita ed esplicita, in cui si pone attenzione al progressivo realizzarsi di forme corrette dell'italiano standard e di enunciati in diverse varietà della lingua. In modo analogo, nel Quadro di Riferimento Invalsi si evidenzia il ruolo rivestito dal percorso di scolarizzazione nel raffinare le competenze grammaticali attraverso la conoscenza e l'uso delle due grammatiche, quella implicita e quella esplicita e, contemporaneamente, «attraverso un uso man mano più ampio, più specifico e più controllato delle diverse varietà linguistiche» ${ }^{6}$.

Entrambi i documenti tengono conto dello sviluppo cognitivo degli apprendenti. Nelle Indicazioni naz̧ionali si pone in evidenza il ruolo della strumentalità della lettura e della scrittura come prima forma di metariflessione che il bambino svolge sulla lingua e si sottolinea la necessità che, nei primi anni di scuola primaria, l'uso della lingua e della riflessione sulla lingua costituiscano un'esperienza inscindibile, evidenziando come «nella pratica coincidano: l'apprendimento della strumentalità del leggere e dello scrivere è da considerarsi infatti un'attività linguistica e metalinguistica al tempo stesso» ${ }^{7}$. Nel Quadro di Riferimento si illustra come, nella realizzazione dei diversi quesiti dell'ambito grammaticale, si tenga conto degli obiettivi curricolari individuati per i diversi gradi di istruzione, diversificando le richieste in base alle capacità cognitive di osservazione, riflessione, astrazione degli allievi ed alle conoscenze che ne derivano.

Gli oggetti d'indagine linguistica da proporre agli apprendenti rappresentano un ulteriore aspetto che i due documenti condividono. Nelle Indicaz̧ioni Narionali

\begin{abstract}
oggetti della riflessione sulla lingua e della grammatica esplicita sono: le strutture sintattiche delle frasi semplici e complesse [...]; le parti del discorso, o categorie lessicali; gli elementi di coesione che servono a mettere in rapporto le diverse parti della frase o del testo (connettivi di vario tipo, pronomi, segni di interpunzione), il lessico e la sua organizzazione; le varietà dell'italiano più diffuse.

(Indicazioni Nazionali del curricolo per l’infanzia e la scuola del primo ciclo, 2012: 39)
\end{abstract}

Nel Quadro di Riferimento il sistema della lingua viene descritto su più livelli: pragmaticotestuale, logico-semantico, morfosintattico, lessicale e fonologico-grafico. Quest'ultimo livello riveste un ruolo a sé, in quanto, se da una parte si sottolinea la necessità che la competenza ortografica debba essere appresa nei primi anni di scolarizzazione, dall'altra si evidenzia anche l'esigenza che essa sia monitorata durante tutti i livelli scolastici. 亡̀ infatti opportuna una ricorsività costante dell'insegnamento/apprendimento dell'ortografia durante l'intero percorso scolastico (De Mauro, 1977b; Serianni, 2015). Questa ciclicità si ritrova nelle stesse consegne dei quesiti Invalsi proposti per i diversi livelli di scolarità, sebbene con differenti livelli di complessità linguistica e cognitiva ${ }^{8}$.

${ }^{6}$ Quadro di Riferimento delle prove Invalsi di italiano (2018: 6).

7 Indicazioni Nazionali del curricolo per l'infanzia e la scuola del primo ciclo (2012: 30).

${ }^{8}$ De Mauro è intervenuto più volte sulla questione ortografica, tentando di ridimensionarne l'importanza ed evidenziando come essa sia appresa dal bambino intorno ai sei, sette anni d'età, momento in cui «padroneggia con notevole abilità» la propria lingua materna, ma considerando anche che le altre facoltà, ad 
Nelle Indicazioni si dedica una sezione specifica all'acquisizione del lessico produttivo e ricettivo in una prospettiva di riflessione linguistica, che si ritrova anche nelle consegne della sezione grammaticale delle prove Invalsi, facendo riferimento soprattutto ai meccanismi linguistici di formazione delle parole ed alle relazioni esistenti tra le famiglie di parole.

Condiviso da entrambi i documenti risulta essere un approccio plurale nei confronti dei differenti modelli teorici di riferimento con cui operare nell'indagine sulla e con la lingua; infatti, nelle Indicarzioni è lasciata all'insegnante la possibilità di scegliere «il modello grammaticale di riferimento che gli sembra più adeguato ed efficace» ${ }^{9}$, possibilità ripresa anche dal Quadro di Riferimento, vista la compresenza di più modelli teorici utilizzati nella scuola; tuttavia, si sottolinea come si sia scelto, nella formulazione dei quesiti, «di fare riferimento, in linea di massima, ai contenuti più noti e condivisi, introducendo però anche alcuni dei contenuti innovativi più assodati nel mondo della ricerca ${ }^{10}$ con l'attenzione a rendere trasparenti le richieste, facendo ricorso ad una terminologia nota, ad esempi o perifrasi esplicative.

Infine, per quanto riguarda la riflessione linguistica e grammaticale, i due documenti sono accomunati da un ulteriore elemento, ossia la modalità con cui osservare, descrivere, analizzare $i$ fatti di lingua. Si raccomanda, infatti, all'insegnante di non limitarsi a far acquisire agli allievi la capacità di memorizzare, riconoscere e denominare le classi e sottoclassi grammaticali, ma, attraverso l'uso di un approccio induttivo, di sviluppare forme di sviluppo critico, promuovendo rielaborazioni e analisi dei fenomeni linguistici osservati, declinati in base all'età degli apprendenti, in modo da favorire il progressivo utilizzo di un metodo scientifico ${ }^{11}$.

\section{Le PROVE INVALSI, LA SCUOLA E LA RIFLESSIONE SULLA LINGUA: UN'INDAGINE ESPLORATIVA IN UN SISTEMA COMPLESSO}

Dopo aver illustrato gli elementi linguistici e didattici che convivono nelle Indicazioni nazionali e il Quadro di Riferimento dell'Invalsi, si rivela interessante domandarsi come siano vissute, percepite ed utilizzate da parte degli insegnanti della scuola primaria le prove somministrate. La finalità dell'indagine è quella di fotografare lo stato dell'arte, con il tentativo di illustrare:

a) il vissuto professionale dei docenti nei confronti delle prove;

b) i legami tra la struttura organizzativa dei diversi istituti scolastici e l'utilizzo degli esiti emersi nei diversi anni in una prospettiva di miglioramento;

c) l'attenzione che i docenti pongono sui contenuti proposti, sulle richieste e gli aspetti della lingua interessati;

d) le ricadute didattiche delle scelte metodologiche adottate nell'insegnamento della grammatica.

esempio la scioltezza espressiva, la padronanza sintattica e la ricchezza lessicale, si sviluppino in modo indipendente «dalla capacità di scrivere in modo ortograficamente corretto». Serianni prosegue evidenziando come il permanere dell'errore ortografico durante l'adolescenza possa rivelarsi indicativo di «scarsa dimestichezza con la lettura e gli automatismi da essa indotti», esponendo l'individuo ad una «forte sanzione sociale». Per tali ragioni, Serianni suggerisce l'opportunità di mantenere un'attenzione sull'«infrazione ortografica» anche nella scuola secondaria di secondo grado (De Mauro, 1977b: 58, 60; Serianni, 2021: 66, 67).

${ }^{9}$ Indicazioni Nazionali del curricolo per l'infanzia e la scuola del primo ciclo (2012: 39).

${ }^{10}$ Quadro di Riferimento delle prove Invalsi di italiano (2018: 10).

11 Indicazioni Nazionali per il curricolo della scuola dell'infanzia e del primo ciclo d'Istruzione (2012: 39) e Quadro di Riferimento Invalsi (2018: 4). 
I dati che si intendono presentare emergono da una ricerca realizzata a partire da un progetto di tesi svolto presso il Corso di Laurea in Scienze della Formazione Primaria dell'Università degli Studi di Torino, nell'anno accademico 2020/2021 ${ }^{12}$.

\subsection{Aspetti metodologici della ricerca}

La ricerca si è svolta nel mese di febbraio 2021con un metodo empirico quantitativo con l'obiettivo di esplorare gli atteggiamenti, le conoscenze e le esperienze professionali dei docenti di scuola primaria del territorio torinese e cuneese rispetto alle Prove Invalsi ed il loro utilizzo nella pratica del miglioramento didattico, con un'attenzione specifica nei confronti della sezione dedicata alla Riflessione sulla lingua. L'indagine si sofferma, inoltre, sulle caratteristiche degli approcci didattici utilizzati dai docenti in questo ambito, ponendo in luce le tipologie di attività proposte per stimolare la riflessione sulla lingua e la quantità di tempo dedicato a ciascun contenuto grammaticale previsto dal Quadro di Riferimento del 2018.

La stesura delle domande del questionario è avvenuta in due modi: nella prima sezione, su aspetti più generali - riguardanti, ad esempio la qualità della collaborazione tra i docenti, l'attenzione della scuola ai bisogni formativi degli insegnanti, il confronto con le famiglie sulle linee educative, l'esistenza o meno di una programmazione comune e di un confronto tra colleghi dello steso ambito disciplinare, la realizzazione efficace dell'inclusione degli studenti con bisogni educativi speciali, le attività che vengono svolte in classe, ecc. - si è scelto di riadattare alcuni quesiti presenti in un'indagine realizzata e condotta dall'Invalsi, all'interno di un progetto PON (Piano Operativo Nazionale) e dell'iniziativa $V A L E S$ (Valutazione e Sviluppo della Scuola), rivolta agli insegnanti dei diversi ordini di scuola nell'anno scolastico $2012 / 2013^{13}$; nella seconda parte, dedicata alla riflessione linguistica, si sono adottati come modelli le proposte contenute nelle Indicarioni Nazionali ed il Quadro di Riferimento Invalsi.

Come strumento di rilevazione indiretta si è utilizzato un questionario condiviso tramite l'applicazione digitale Moduli Google, inviando il collegamento al link, con l'ausilio della posta elettronica, ai dirigenti scolastici degli Istituti Comprensivi del territorio cuneese e della città di Torino; $i$ docenti hanno avuto la possibilità di compilare il questionario nel mese di febbraio 2021. Si tratta, quindi, di un campione non probabilistico, costituito in base alla disponibilità dei soggetti a partecipare all'indagine.

Il questionario è composto da diciotto domande che si succedono, seguendo una visione dal generale al particolare. Le domande sono di diverso tipo: dicotomiche, a scelta multipla, a testo libero, con scala di giudizio numerico e con scala di giudizio grafica. La tabella seguente ha lo scopo di illustrare la struttura del questionario, evidenziando i campi di indagine e le variabili, in relazione al numero dei quesiti corrispondenti.

Figura 1. Descrizione delle domande del questionario

\begin{tabular}{|l|l|c|}
\hline \multicolumn{1}{|c|}{ Aree da indagare } & \multicolumn{1}{|c|}{ Variabili } & Quesiti \\
\hline \multirow{4}{*}{ Variabili di sfondo } & Genere & 1 \\
\cline { 2 - 3 } & Età & 2 \\
\cline { 2 - 3 } & Istruzione e professione & $3-4-5-6$ \\
\cline { 2 - 3 } & Caratteristiche Istituti scolastici & $7-8-12$ \\
\hline
\end{tabular}

${ }^{12}$ La tesista che ha contribuito all'attuazione della ricerca è Sara Gallo, con una tesi dal titolo Contrastare l'analfabetismo funzionale: riflettere sulla lingua a partire dalle prove INV ALSI, a.s. 2020/2021.

13 https://www.invalsi.it/invalsi/ri/vales/doc.php. 
(C) Italiano LinguaDue 2. 2021. M. Roccia, Le prove Invalsi e la riflessione sulla lingua nella scuola primaria: un'indagine sulla percezione degli insegnanti

\begin{tabular}{|l|l|c|}
\hline Rilevazioni INVALSI & Competenza ed esperienza professionale e INVALSI & 9-10-11-14 \\
\cline { 2 - 3 } & $\begin{array}{l}\text { Percezione ed atteggiamenti rispetto alle prove e agli } \\
\text { esiti delle indagini INVALSI }\end{array}$ & $13-15$ \\
\hline Riflessione linguistica & $\begin{array}{l}\text { Percezione e conoscenze professionali rispetto alla } \\
\text { sezione di Riflessione linguistica contenuta nelle } \\
\text { prove INVALSI }\end{array}$ & 16 \\
\cline { 2 - 3 } & $\begin{array}{l}\text { Scelte didattiche, metodologiche e di contenuto } \\
\text { rispetto alla Riflessione linguistica }\end{array}$ & $17-18$ \\
\hline
\end{tabular}

\subsection{Esiti della ricerca: macroaree di interesse}

La scelta del questionario come strumento di rilevazione dei dati è finalizzata a raggiungere il maggior numero possibile di insegnanti di scuola primaria e ad avere così un quadro ampio e significativo, seppur in un territorio circoscritto. La partecipazione dei docenti è stata considerevole e ha permesso la costituzione di un campione formato da 163 persone, in cui il $74,20 \%$ del totale è rappresentato da insegnanti di istituti del territorio cuneese ed il restante $25,80 \%$ da insegnanti di istituti della città di Torino.

Per quanto concerne i dati anagrafici e professionali, emerge una netta maggioranza di docenti di genere femminile, pari al 96,90\% del totale dei partecipanti, la cui età media risulta essere di 47 anni (l'87,10\% ha più di 36 anni). Il titolo di studio più rappresentativo è il diploma di scuola superiore $(51,20 \%)$, con un numero altrettanto consistente di laureati in Scienze della Formazione Primaria, pari al 25,20\% del campione, seguito da altri tipi di laurea, soprattutto di indirizzo pedagogico ed umanistico. Appare interessante che circa la metà del campione, il 51,2\% del totale, svolga la propria attività professionale nel medesimo istituto da più di dieci anni e che il 31,9\% abbia avuto esperienze professionali in più di un istituto. Questi dati si mostrano utili perché, nel primo caso, si tratta di insegnanti che hanno una conoscenza approfondita dell'istituto in cui operano e, nel secondo, di docenti con esperienze in realtà differenti.

Dai dati generali evidenziati, emergono esiti e spunti di riflessione che si possono enucleare in cinque macrotemi:

1. l'organizzazione di un istituto scolastico come sistema complesso;

2. la percezione rispetto all'inclusione dei bisogni educativi;

3. l'utilizzo delle prove Invalsi e degli esiti relativi nella progettazione didattica d'istituto curricolare in un'ottica di miglioramento;

4. le finalità e gli obiettivi generali dell'Invalsi e, in particolare, per la riflessione linguistica;

5. le scelte metodologico didattiche utilizzate nell'insegnamento della riflessione linguistica ed i contenuti proposti nel percorso didattico.

\section{LE PROVE INVALSI E L'ORGANIZZAZIONE DI UN SISTEMA COMPLESSO: GLI ESITI DELLA RICERCA}

\subsection{L'organizzazione di un istituto scolastico come sistema complesso}

Per quanto riguarda l'organizzazione di un istituto scolastico come sistema complesso, si evidenzia (Figura 2) come, esista una Commissione Invalsi nel 58,3\% delle scuole che 
hanno partecipato all'indagine, non presente, invece, nel restante 41,7\%. La Commissione Invalsi ha lo scopo di occuparsi dell'organizzazione della somministrazione delle prove, della rielaborazione degli esiti ottenuti dal singolo istituto, della comunicazione ai docenti dei risultati per agire in una prospettiva di miglioramento complessivo, e di indirizzare le loro scelte sia nella progettazione e nell'azione didattica da intraprendere sia nella valutazione di percorsi di aggiornamento e formazione per $\mathrm{i}$ docenti in servizio. L'obiettivo primario della Commissione Invalsi dovrebbe essere dunque quello di orientare, sulla base delle evidenze, l'operato dei docenti, legando tra loro anche i diversi ordini di scuola verso una prospettiva comune.

Figura 2. Risultati in percentuale domanda 9

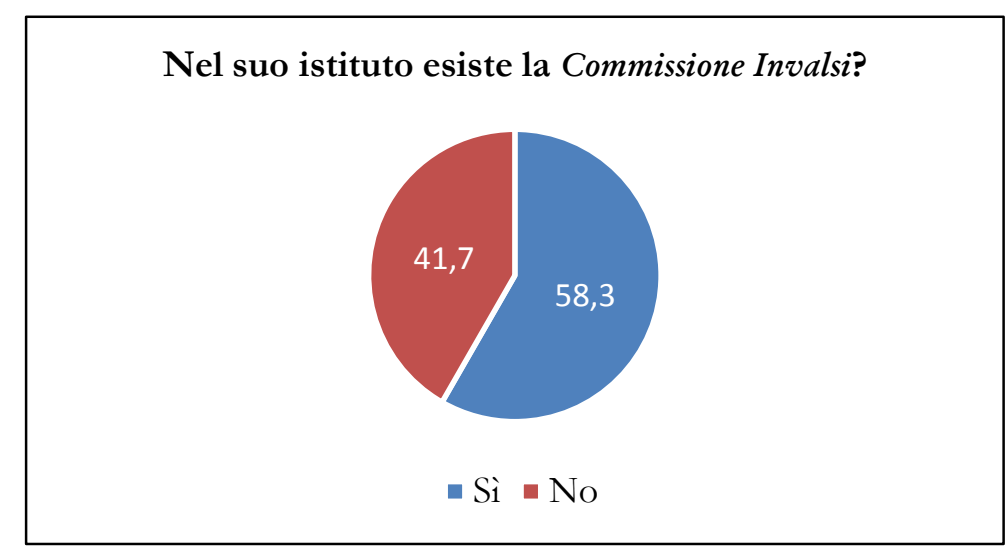

Tuttavia, seppur nelle scuole sia presente una Commissione Invalsi, solo nell' $86 \%$ dei casi questa Commissione (Figura 3) fornisce al Collegio docenti i dati rielaborati rispetto agli esiti ottenuti dagli allievi nelle prove stesse. I dati rilevati dalle prove risulterebbero essere elementi determinanti per progettare un'azione didattica atta al progressivo potenziamento delle competenze grammaticali e di lettura degli allievi, rispondendo così a quello che rappresenta l'obiettivo prioritario perseguito dall'Invalsi, ossia il miglioramento delle competenze degli studenti. Perché questo si realizzi è necessario che i docenti abbiano a disposizione gli strumenti (e le prove Invalsi lo sono) per individuare su quali processi e fasi dell'insegnamento/apprendimento della lettura e della grammatica si debba intervenire attivamente.

Figura 3. Risultati in percentuale domanda n. 10

Se sì, la Commissione rielabora $\mathrm{i}$ risultati e procede a una comunicazione descrittiva utile al Collegio docenti come prospettiva di azioni di miglioramento didattico?

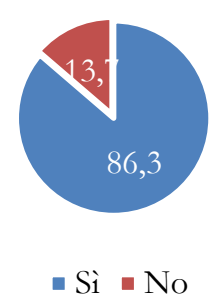


In un'approfondita valutazione sulle evidenze emerse dagli esiti delle prove Invalsi, Alberto Sobrero (2020: 138) indica che cosa debbano fare le scuole per utilizzare in modo proficuo i dati rintracciati:

[...] la valutazione finalizzata al miglioramento richiede il concorso di almeno cinque fattori: contesto e risorse; esiti interni (giudizi d'aula); risultati INVALSI; analisi delle prove e dei livelli; autovalutazione comparativa di tipo quali-quantitativo (confronto dei risultati con quelli degli anni precedenti). Il miglioramento che voglia partire dall'utilizzazione al meglio delle opportunità offerte dai risultati della valutazione (Faggioli, 2014) dovrà dunque stabilire una relazione fra i risultati INVALSI e la didattica in atto, attraverso l'analisi qualitativo-quantitativa comparativa, che passa fra almeno quattro fasi:

1. analisi del Report di scuola (autovalutazione RAV);

2. analisi delle prove e dei risultati;

3. analisi degli apprendimenti (in riferimento ai Quadri di Riferimento, alle Indicazioni Narionali, ai documenti di programmazione, alle esperienze e sperimentazioni didattiche, alla descrizione delle prove, utilizzando anche le Guide alla lettura e i Quaderni del Sistema Nazionale di Valutazione (reperibili nel sito INVALSI);

4. riflessione critica sulle pratiche didattiche e valutative usate in classe per determinare tali apprendimenti.

È a questo punto che sarà possibile costruire un progetto fondato e ben motivato di azioni migliorative, che coinvolga il maggior numero di discipline: terrà conto dei lati positivi e delle criticità delle pratiche didattiche, e riguarderà tanto i processi quanto i risultati [...].

Di fronte a queste indicazioni, appare ancora più interessante scandagliare gli esiti dell'indagine circa l'organizzazione del sistema.

Figura 4. Risultati in percentuale domanda n.12_B

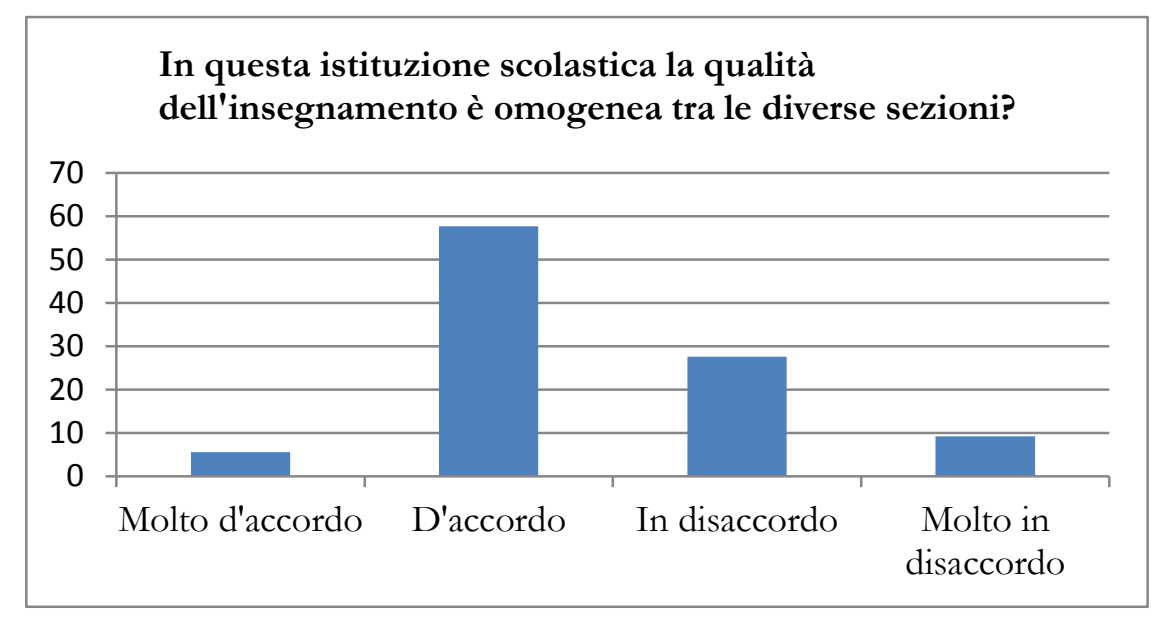

Dalle risposte al quesito "In questa istiturione scolastica, la qualità dell'insegnamento è omogenea tra le diverse sezioni?" (Figura 4) emerge come il 36,8\% (somma che rappresenta le percentuali totali delle risposte disaccordo e molto in disaccordo) dichiari di percepire una certa disomogeneità tra le proposte attuate nelle diverse classi dei vari istituti; quindi, si rivela opportuno cercare di comprendere quali siano le azioni utilizzate per permettere un confronto tra i docenti nella progettazione relativa sia all'istituto nel suo complesso sia curricolare per disciplina. 
(C) Italiano LinguaDue 2. 2021. M. Roccia, Le prove Invalsi e la riflessione sulla lingua nella scuola primaria: un'indagine sulla percezione degli insegnanti

Figura 5. Risultati in percentuale domanda n.12_G

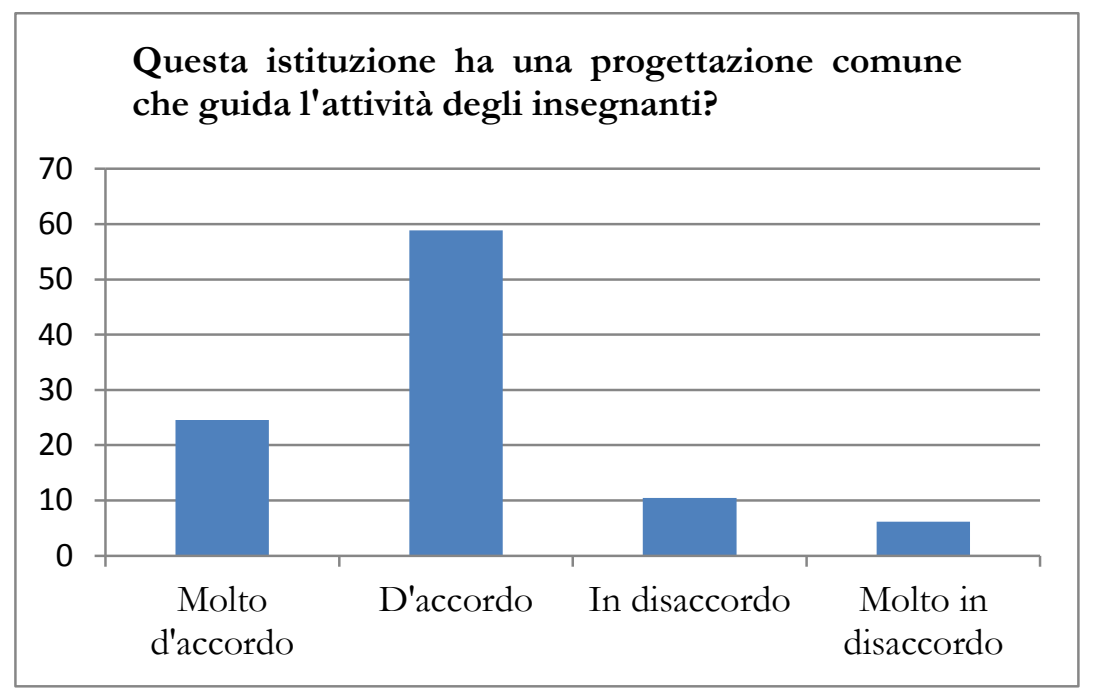

Alla domanda "Questa istiturione ha una progettazione comune che guida l'attività degli insegnanti?' (Figura 5) 1'83,44\% (somma che rappresenta le percentuali totali delle risposte d'accordo e molto d'accordo) dichiara che esiste una progettazione disciplinare d'istituto comune ai diversi ordini di scuola. Tuttavia se si approfondisce ulteriormente l'argomento con la domanda "In questa istiturione i collegbi dello stesso ambito disciplinare si confrontano durante l'anno scolastico?" (Figura 6) si evidenzia come nel 22,7\% dei casi (somma che rappresenta le percentuali totali delle risposte in disaccordo e molto in disaccordo) non vi sia uno scambio diretto con gli altri docenti della medesima disciplina che operano all'interno dello stesso istituto. Rispetto ai dati complessivi, emerge che nel 6,1\% dei casi non è presente né una progettazione disciplinare redatta e riconosciuta come condivisa da tutto l'istituto scolastico, né vi è la possibilità per gli insegnanti di partecipare, durante l'anno scolastico, a momenti istituzionalizzati dedicati all'interazione e allo scambio reciproco.

Figura 6. Risultati in percentuale domanda 12_F

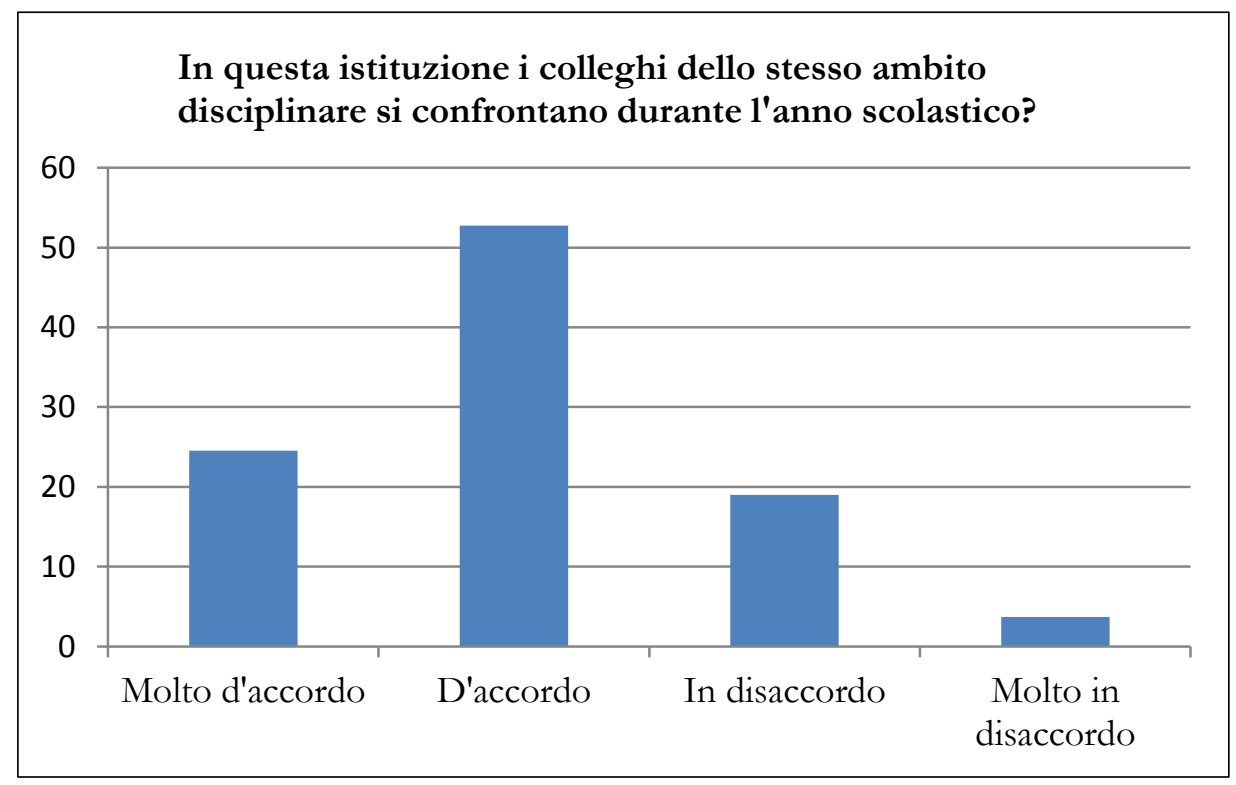


(C) Italiano LinguaDue 2. 2021. M. Roccia, Le prove Invalsi e la riflessione sulla lingua nella scuola primaria: un'indagine sulla percezione degli insegnanti

\subsection{La percezione rispetto all'inclusione dei bisogni educativi}

Figura 7. Risultati in percentuale domanda n. 12_H

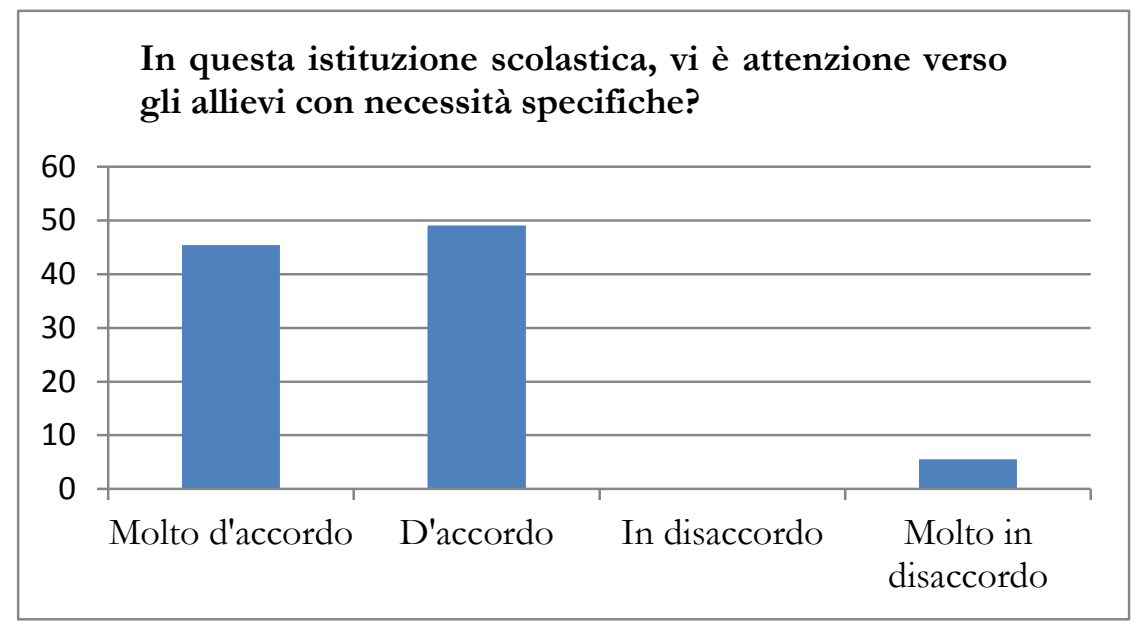

Per quanto riguarda le singole istituzioni scolastiche e l'inclusione degli allievi che mostrano bisogni educativi speciali, sono state poste due domande per comprendere la percezione dei docenti rispetto alle azioni didattiche e formative attuate nelle particolari situazioni di necessità; il primo quesito ha un carattere più generale, mentre il secondo intende indagare un aspetto specifico.

Alla domanda "In questa istituzione scolastica, vi è attenzione verso gli allievi con necessità specifiche?'(Figura 7) le risposte in cui i docenti si dichiarano molto d'accordo e d'accordo risultano essere rispettivamente del $45,4 \%$ e del $49,1 \%$; solo il 5,52\% risponde molto in disaccordo. Dai dati risulta come, nella quasi totalità delle scuole dei docenti che hanno risposto alla domanda, si presti attenzione a proporre percorsi personalizzati ed individualizzati in base alle diverse necessità didattiche e formative degli allievi con BES.

Tuttavia, alla domanda riguardante una particolare tipologia di allievi con BES "In questa istituzione scolastica, si realizza in modo efficace l'inclusione per gli allievi non italofoni?" (Figura 8) le percentuali cambiano: infatti, la risposta molto d'accordo decresce al $24,54 \%$ e si amplia la percentuale di coloro che dichiarano di essere in disaccordo e molto in disaccordo, raggiungendo il 12, $27 \%$ del totale.

Figura 8. Risultati in percentuale domanda n. 12_H

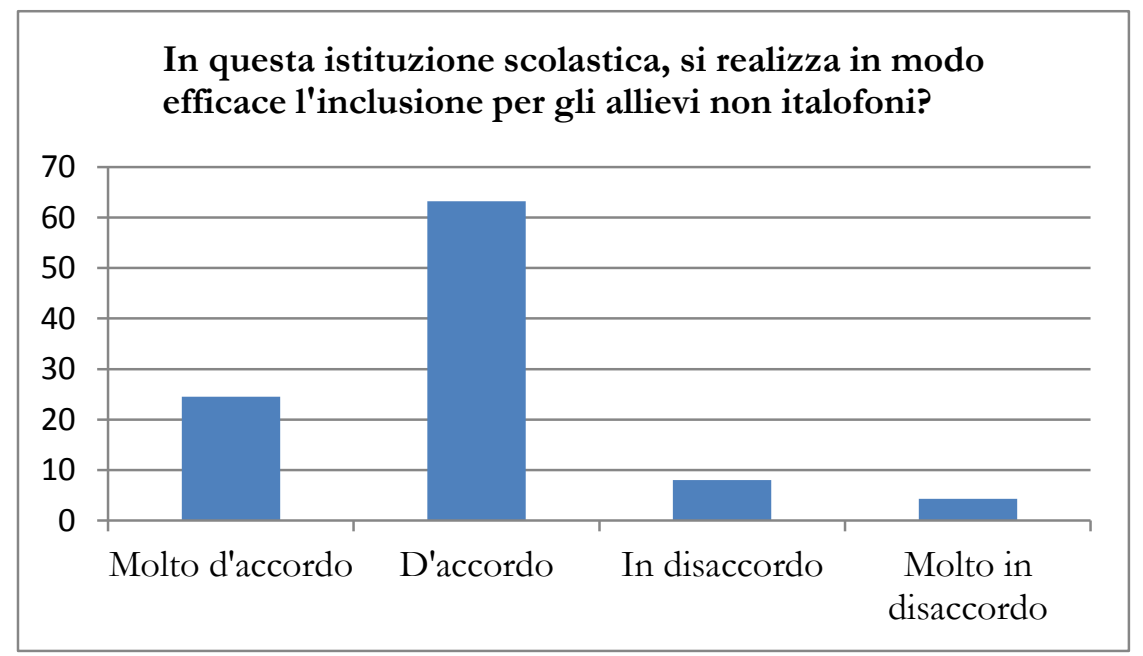


Sembra dunque che, in alcune scuole dei rispondenti, non emerga la necessità di operare attivamente nella realizzazione di progetti finalizzati all'apprendimento dellitaliano come lingua seconda e di percorsi di insegnamento indirizzati specificatamente agli allievi neoarrivati o di seconda generazione, malgrado i risultati ottenuti dagli studenti nativi e non nativi nelle prove somministrate dall'Invalsi - come risulta dalla Figura 9, in cui si illustrano gli esiti nazionali per le varie discipline al termine della scuola primaria nell'anno scolastico 2020/2021 - mostrino come gli allievi di origine straniera abbiano ottenuto punteggi inferiori in italiano e in matematica (ma non in inglese) rispetto ai nativi.

Figura 9. Esiti test Invalsi a.s. 2020/2021_allievi non italofoni

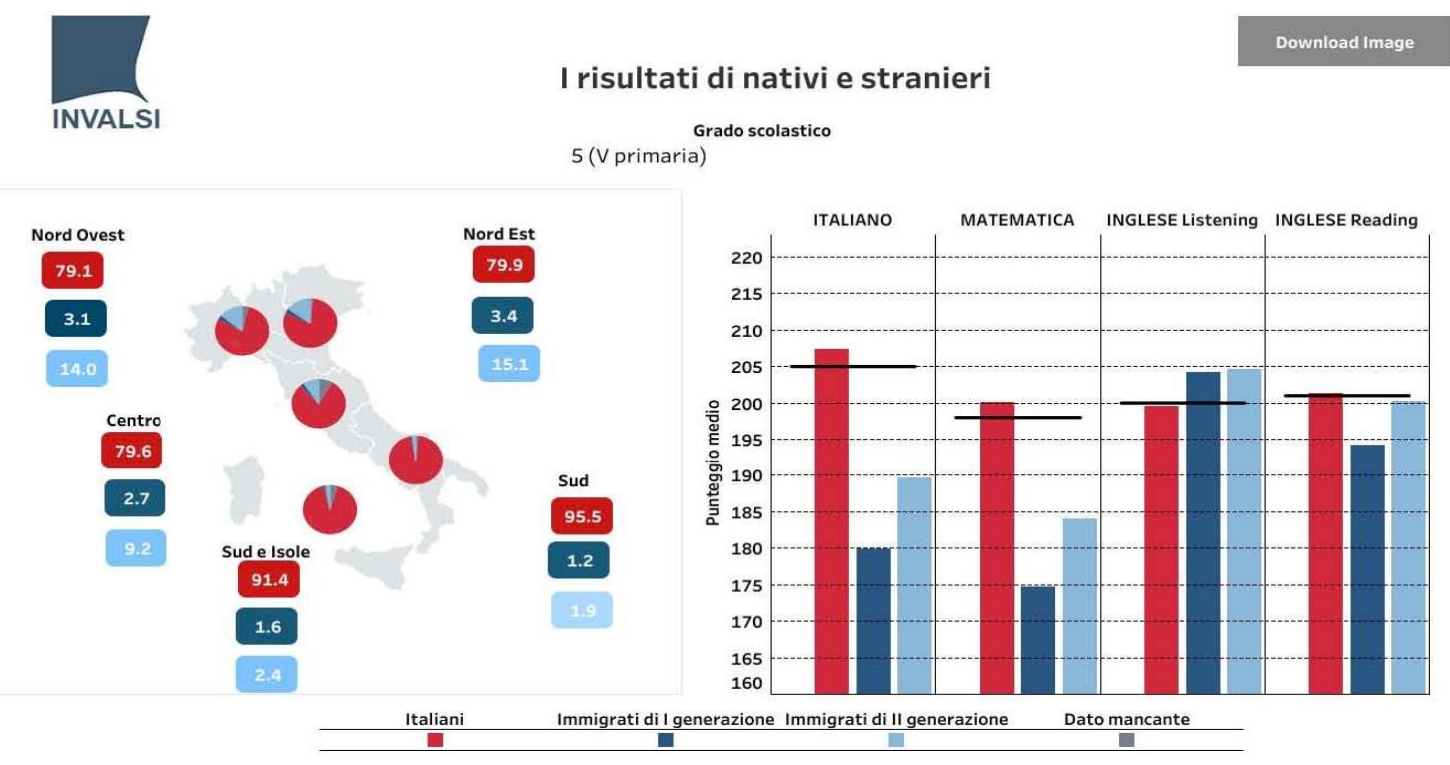

(Fonte: Invalsi open, Sito ufficiale area prove nazionali, I risultati delle Prove INVALSI 2021: https://public.tableau.com/app/profile/invalsi/viz/RAPPORTO2020-

2021_16261728450410/INIZIO)

\subsection{L'utilizæo delle prove Invalsi e degli esiti relativi nella progettazione didattica d'istituto e curricolare in un'ottica di miglioramento}

In questa sezione sono esposti e analizzati i dati relativi al terzo macrotema della nostra indagine, ossia la relazione esistente tra l'utilizzo delle prove dell'Invalsi, i relativi esiti e la progettazione didattica, sia d'istituto sia curricolare, in una prospettiva di sviluppo. La domanda numero 13 richiedeva, in base alla propria esperienza professionale, di esprimere il livello di accordo o disaccordo riguardo alcune problematiche inerenti il legame tra i contenuti proposti, i risultati ottenuti dagli studenti nelle prove Invalsi e la ricaduta di tali esiti nella la realtà scolastica specifica.

Per quanto riguarda il primo punto del tredicesimo quesito, ossia se le procedure di somministrazione delle prove siano comunicate dall'ente in modo chiaro e comprensibile, l'83,43\% degli insegnanti risponde di essere molto d'accordo e d'accordo; al secondo punto, in cui si richiedeva se i tempi di restituzione dei dati siano adeguati, risposte positive sono date dal 61,96\% del campione; il 38,04\% dichiara di non essere soddisfatto dei tempi trascorsi tra la somministrazione delle prove e la conoscenza dei risultati. Al terzo punto, "I risultati delle prove restituiti alle scuole sono chiari e comprensibili?" si evidenzia un riscontro 
positivo per il 75,46\% $\%$ del campione e il 73,62\% risponde che le informazioni riguardanti le prove (Quadri di riferimento, Guida alla Lettura) sono facilmente reperibili e fruibili.

Se nel primo punto della domanda 13 del questionario l'intento è quello di comprendere come i docenti percepiscono modalità, procedure, tempi e qualità delle informazioni prodotte dall'Invalsi, nella seconda sezione di questa domanda si pone l'attenzione sul rapporto reale tra gli esiti ricevuti ed il loro utilizzo nell'istituto e nell'attività didattica dei docenti. In questo secondo caso, la finalità è quello di rilevare come i risultati forniti abbiano una ricaduta nella pratica scolastica e quale ruolo rivestano nella progettazione di azioni didattiche finalizzate al miglioramento delle competenze degli allievi.

Figura 10. Risultati in percentuale domanda 13_punto 6

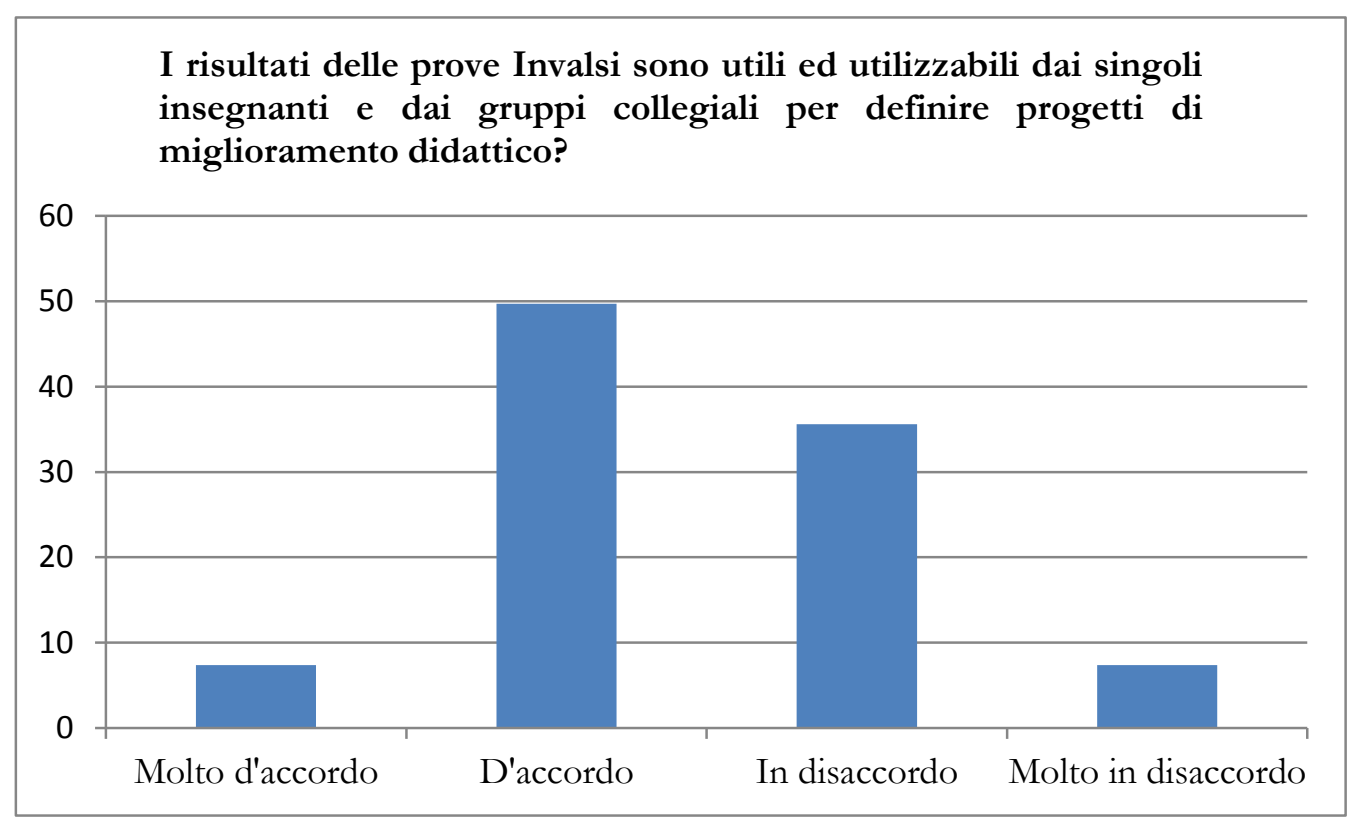

I dati raccolti evidenziano una certa contraddizione con le risposte fornite al primo punto della domanda 13. Come si evince dalla Figura 10, in cui sono rappresentati gli esiti relativi alla domanda "I risultati delle prove Invalsi sono utili ed utilizzabili dai singoli insegnanti e dai gruppi collegiali per definire progetti di miglioramento didattico?", il 57,06\% dei docenti dichiara di essere molto d'accordo o d'accordo, mentre il 42,94\% risponde di essere in disaccordo o molto in disaccordo, nonostante il $75,46 \%$ degli insegnanti abbia risposto al terzo punto (I risultati delle prove restituiti alle scuole sono chiari e comprensibili?") esprimendo soddisfazione circa la chiarezza dei risultati inviati dall'Invalsi. Ciò significa che percepire i risultati come poco utili per migliorare il proprio percorso e la propria progettazione didattica non è da ricercare nella difficoltà di reperire le informazioni o in ostacoli presenti nella loro rilettura e interpretazione. L'analisi delle risposte alle successive domande del questionario consentirà di formulare alcune ipotesi sul perché gli esiti delle prove forniti dall'Invalsi, nonostante siano giudicati essere chiari e comprensibili, siano ritenuti da quasi la metà del campione di poca utilità nella loro pratica didattica, nell'ottica di un suo possibile miglioramento.

Nel quesito 13 al punto 7, si domanda se la progettazione didattica utilizzata durante l'anno scolastico risponda in modo adeguato agli obiettivi ed alle competenze descritte nelle Indicaz̧ioni Naz̧ionali (Figura 11). 
(C) Italiano LinguaDue 2. 2021. M. Roccia, Le prove Invalsi e la riflessione sulla lingua nella scuola primaria: un'indagine sulla percezione degli insegnanti

Figura 11. Risultati in percentuale_domanda 13 _punto 7

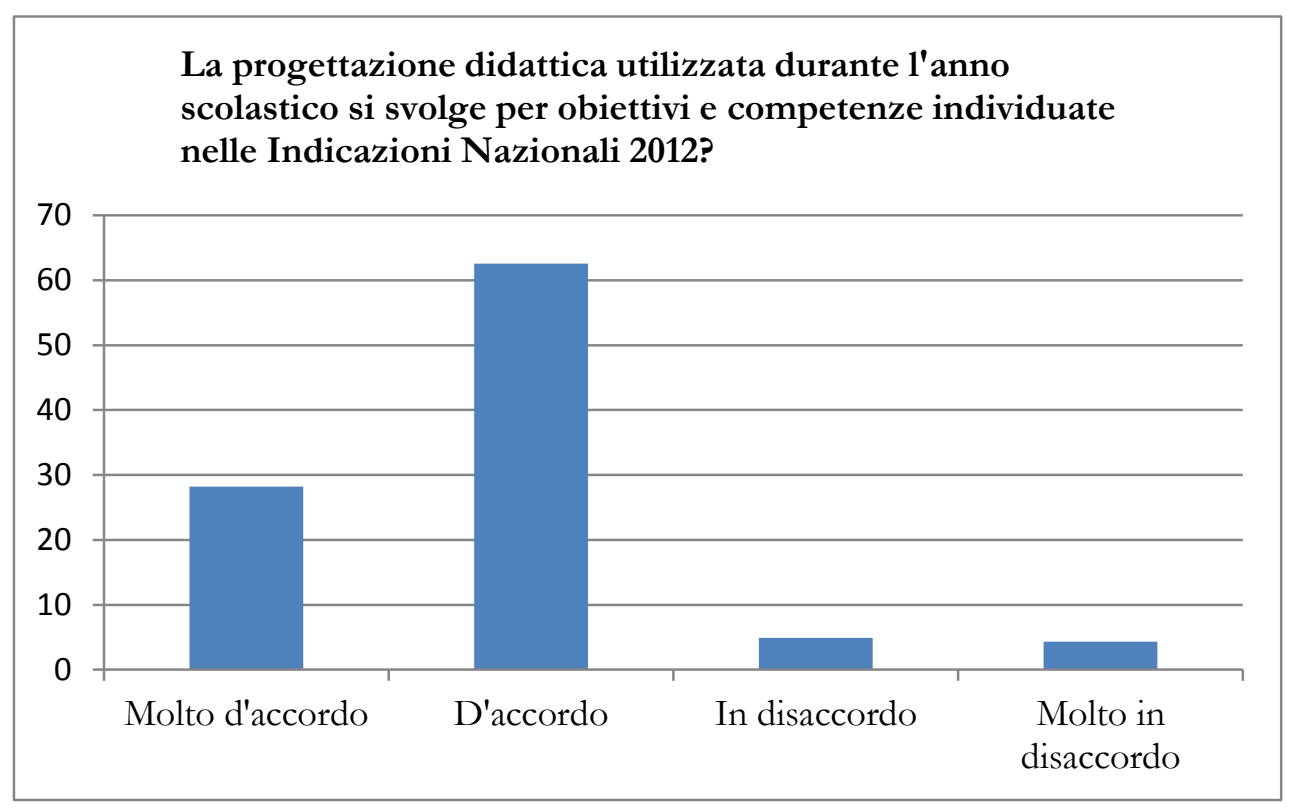

Le risposte positive, ossia la scelta delle opzioni molto d'accordo e d'accordo, sono date dal 90,80\% del campione complessivo; quasi la totalità degli insegnanti intervistati dichiara quindi di svolgere una didattica seguendo le indicazioni proposte nel documento ministeriale.

Figura 12. Risultati in percentuale domanda 13_punto 8

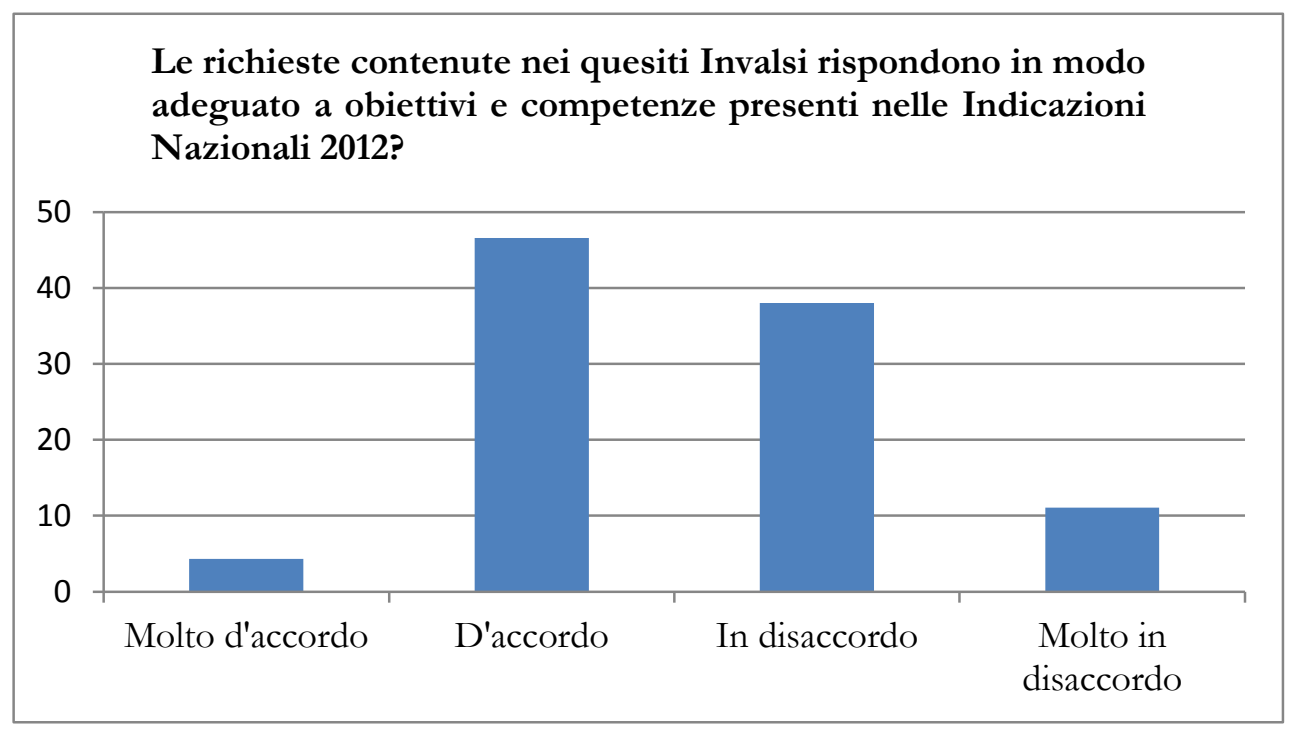

Per rilevare se gli insegnanti percepiscano una correlazione tra gli obiettivi educativi e i traguardi per lo sviluppo delle competenze stabiliti dalle Indicazioni Nazionali e le prove Invalsi si è chiesto ai docenti se "Le richieste contenute nei quesiti Invalsi rispondono in modo adeguato a obiettivi e competenze presenti nelle Indicazioni Nazionali 2012?’. Le percentuali delle risposte (Figura 12) sono così distribuite: 50,92\% molto d'accordo e d'accordo, 49,08\% in disaccordo $\mathrm{o}$ molto in disaccordo. 
Circa la metà del campione ritiene, quindi, che non vi sia una coerente relazione tra le Indicazioni Nazionali e le prove Invalsi, sebbene dalle risposte fornite alla domanda 13, nel punto 4 (Figura 13) si evidenzi la facilità di reperire le informazioni necessarie dal Quadro di Riferimento o dalle Guide alla lettura dei dati, documenti realizzati dall'ente di ricerca come ulteriori strumenti di approfondimento alle prove.

Figura 13. Risultati in percentuale domanda 13 _punto 4

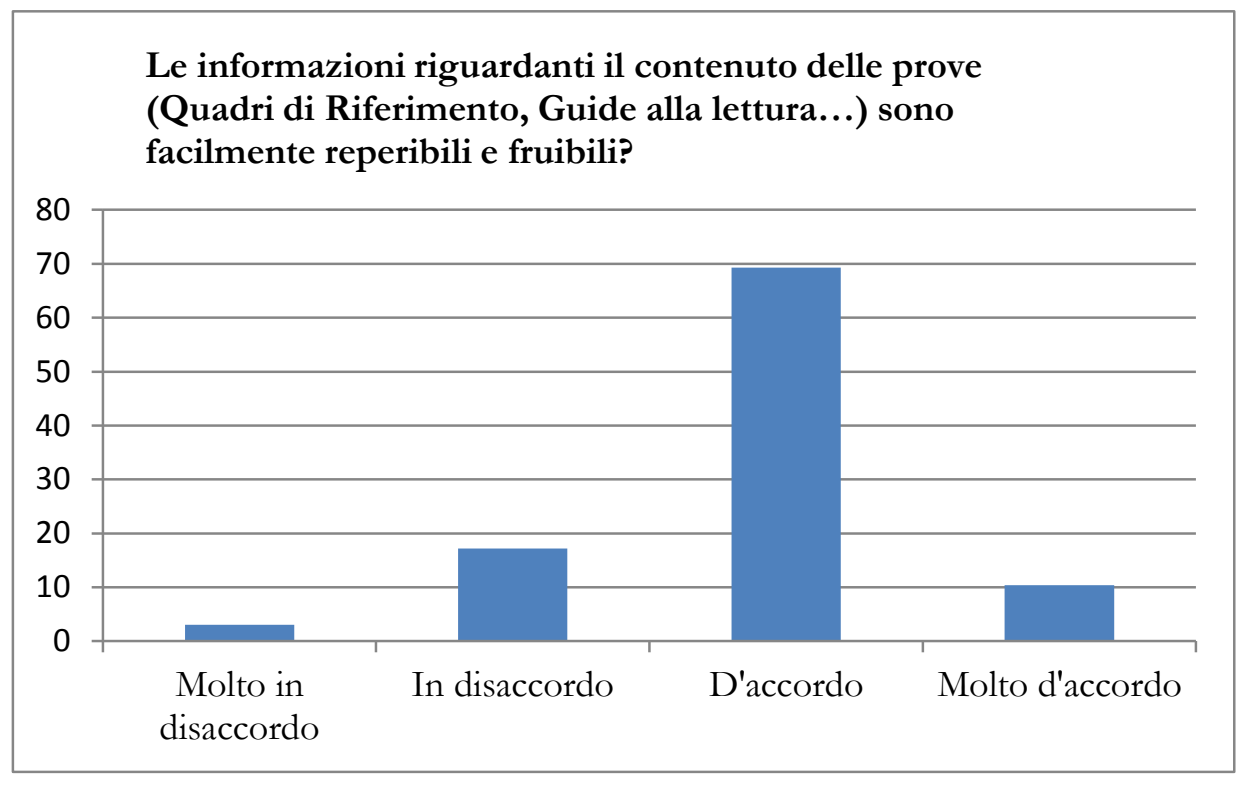

Tornando all'organizzazione di sistema, si è rilevato che il 22,7\% (Figura 6) non si confronta sulle discipline comuni durante l'anno scolastico. L'assenza o la carenza di un dialogo tra i docenti rappresenta un ostacolo che impedisce di operare con efficacia sui processi e sull'azione didattica, attraverso la condivisione dell'analisi dagli esiti forniti dall'Invalsi alla propria scuola. È anche emerso (Figura 10) che il 42,94\% dei docenti percepisce i risultati delle valutazioni Invalsi poco utili ad attuare azioni di miglioramento, in quanto vissuti come non legati alla realtà scolastica, e ritiene che le proposte in esse contenute siano distanti dalle Indicaz̧ioni naz̧ionali, quindi dagli obiettivi e dai traguardi a cui tendere nella progettazione didattica.

Per concludere il terzo macrotema emerso dall'indagine, ossia come i docenti percepiscano l'utilizzo delle prove Invalsi e dei relativi esiti nella progettazione curricolare e didattica d'istituto, si è chiesto loro (quesito 13 al punto 9, Figura 14) di esprimere il proprio grado di accordo o disaccordo rispondendo alla domanda "Le prove Invalsi rispondono in modo adeguato agli obiettivi ed alle competenze presenti nella progettazione d'istituto o di classe?'. Le risposte molto d'accordo e d'accordo rappresentano il 39,26\% del totale, mentre le risposte in disaccordo e molto in disaccordo il 60,74\%. Confrontando le risposte a questa domanda con quelle fornite alla domanda 13, punto 7 (Figura 11), 8 (Figura 12) e 9 (Figura 14), in cui si sondava l'attinenza della progettazione d'istituto e curricolare con le linee presenti nelle Indicazioni Nazionali, la percezione del legame tra le prove Invalsi e le Indicazioni Nazionali e l'interdipendenza tra le prove Invalsi e le competenze contenute nella progettazione curricolare d'istituto, si può notare un progressivo incremento nella distanza avvertita dagli insegnanti tra le varie proposte, fino a giungere a quasi il $61 \%$ del campione (99 docenti su 163) che dichiara di non riconoscere nessi tra i contenuti delle prove Invalsi e quelli proposti nella loro attività scolastica. 
(C) Italiano LinguaDue 2. 2021. M. Roccia, Le prove Invalsi e la riflessione sulla lingua nella scuola primaria: un'indagine sulla percezione degli insegnanti

Figura 14. Risultati in percentuale domanda 13 _punto 9

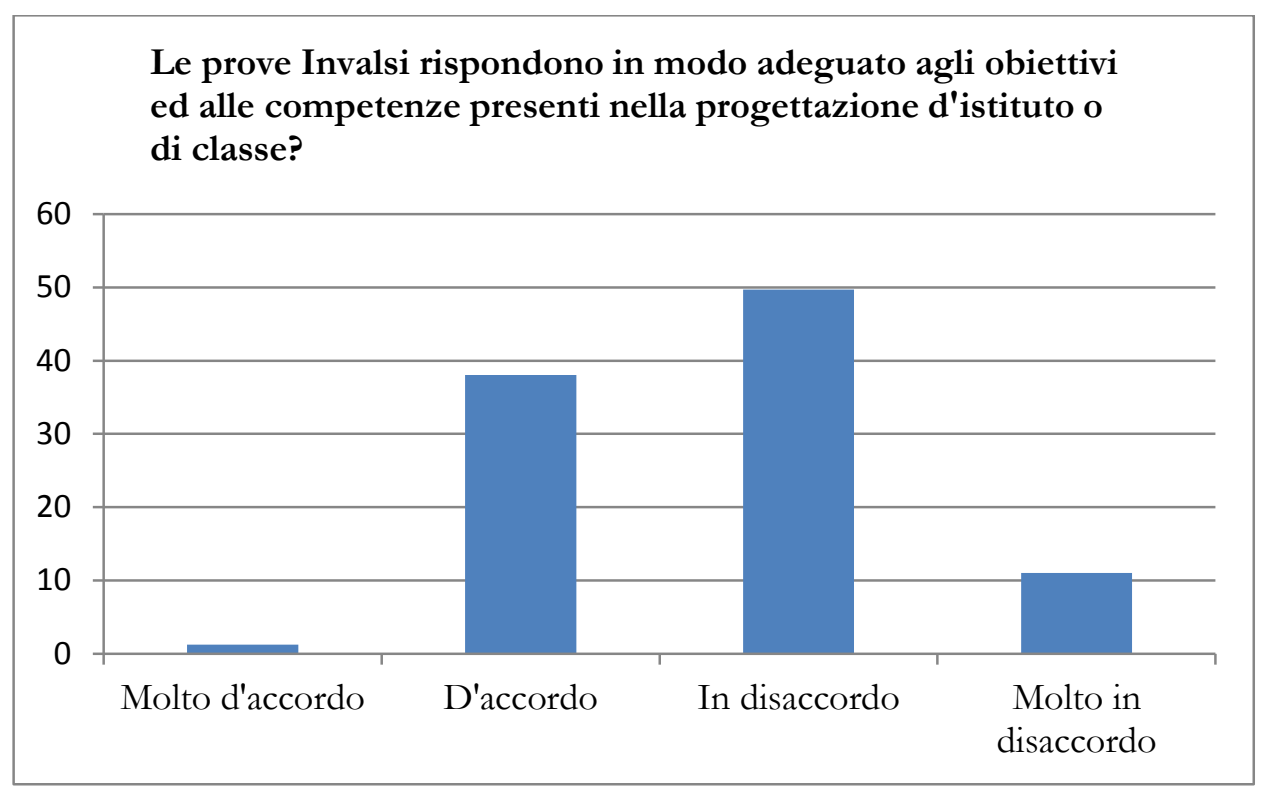

Tuttavia, anche se il 90,80\% del campione ha risposto in modo positivo alla domanda 13 al punto 7 (Figura 11), in cui si chiedeva ai docenti se gli obiettivi e i traguardi di apprendimento sottesi alla loro alla loro azione didattica rispondessero alle linee di lavoro contenute nelle Indicazioni, il 49,08\% del totale afferma di non cogliere una relazione tra le consegne dell'Invalsi e gli obiettivi ed i traguardi illustrati nelle Indicazioni nazionali (Figura 12), e il $60,74 \%$ degli insegnanti dichiara che non vi è corrispondenza tra le richieste contenute nelle prove Invalsi e la progettazione didattica e curricolare d'istituto (Figura 13). Nella percezione degli insegnanti, quindi, emerge un graduale incremento della distanza avvertita tra i documenti ministeriali di riferimento per la scuola, i contenuti previsti dalle prove Invalsi, le progettazioni realizzate e condivise negli istituti e la pratica didattica quotidiana.

Tale distanza risulta maggiore se si considera che quasi il 23\% del campione afferma che non vi sia possibilità di confronto regolare, durante l'anno scolastico, tra colleghi della medesima disciplina (Figura 6) e che quasi il 43\% dichiara che i risultati offerti dalle prove Invalsi non sono utili e fruibili dai docenti per migliorare la loro azione didattica(Figura 10).

5. LE PROVE INVALSI E LA RIFLESSIONE LINGUISTICA NELLA SCUOLA PRIMARIA: GLI ESITI DELLA RICERCA

5.1. Le finalità e gli obiettivi delle prove Invalsi: uno sguardo generale e particolare sulla riflessione linguistica

Nel questionario erano presenti due domande che avevano lo scopo di cogliere come gli insegnanti percepiscano le finalità e gli obiettivi dell'Invalsi, sia in generale sia rispetto alla riflessione linguistica.

Il quesito numero 14 era così formulato: "In base alle sue conoscenze ed alla sua esperienza professionale, quali pensa siano le finalità delle rilevarioni Invalsi?” ed era possibile scegliere tre risposte tra le varie possibilità date (Figura 15). 
(C) Italiano LinguaDue 2. 2021. M. Roccia, Le prove Invalsi e la riflessione sulla lingua nella scuola primaria: un'indagine sulla percezione degli insegnanti

Figura 15. Risultati in percentuale domanda 14

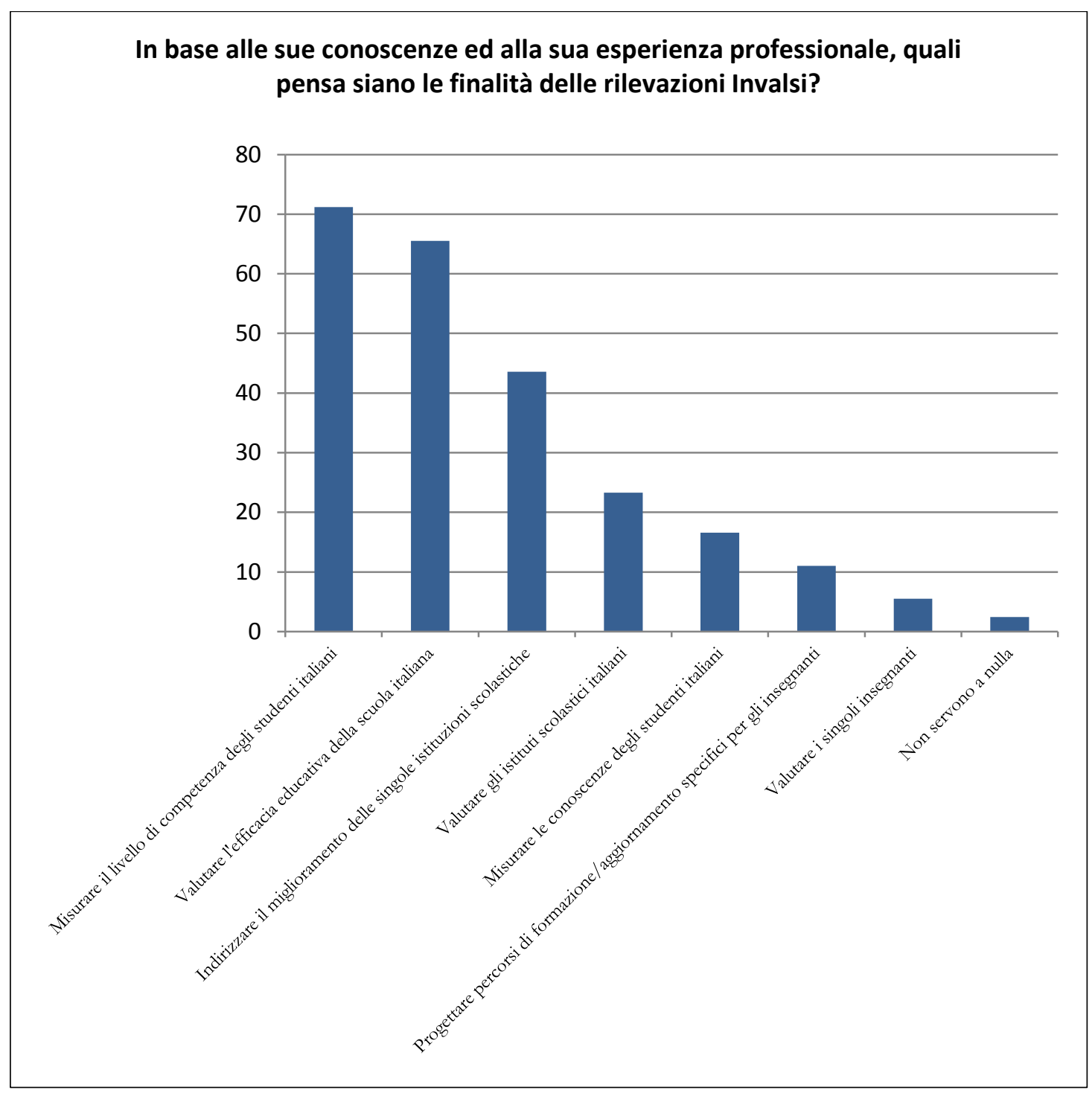

L'opzione per "Misurare il livello di competenza degli studenti italiani” è stata selezionata dal 71,2\% degli insegnanti; "Valutare l'efficacia educativa della scuola italiana" dal $65,6 \%$ e, infine, "Indirizzare il miglioramento della singola istituzione scolastica sulla base delle fragilità rilevate" dal 43,6\%.

Anche in questo caso, si assiste ad una discrepanza in ciò che gli insegnanti dichiarano: infatti, il 65,6\% risponde che tra le finalità dell'Invalsi vi è quella di valutare l'efficacia formativa della scuola; il 43,6\% del campione risponde che, sulla base degli esiti ottenuti, uno degli obiettivi delle prove è quello di indirizzare il miglioramento della qualità formativa e didattica delle singole istituzioni scolastiche per proporre possibili soluzioni agli elementi di fragilità emersi nell'ambito delle discipline di cui l'Invalsi valuta le competenze degli studenti (Figura 15). Tuttavia, come si è illustrato in precedenza, alla domanda 13, punto 6 (Figura 13), il 42,94\% degli insegnanti ha risposto che i risultati ottenuti con la somministrazione delle prove Invalsi non sono utili ed utilizzabili per migliorare il loro insegnamento.

Una conferma della distanza che molti insegnanti avvertono tra la propria azione di insegnamento (approcci, metodi, attività, pratiche didattiche, modalità e tecniche di valutazione, ecc.) e le prove Invalsi si ha se si osservano le percentuali relative ad una delle 
(C) Italiano LinguaDue 2. 2021. M. Roccia, Le prove Invalsi e la riflessione sulla lingua nella scuola primaria: un'indagine sulla percezione degli insegnanti

risposte date alla domanda 15, punto 8 (figura 16), "I risultati mi daranno un quadro oggettivo del livello della mia classe": il 47,24\% del campione risponde di pensarlo talvolta, e il $31,90 \%$ dichiara di non pensarlo mai.

Figura 16. Risultati in percentuale domanda 15

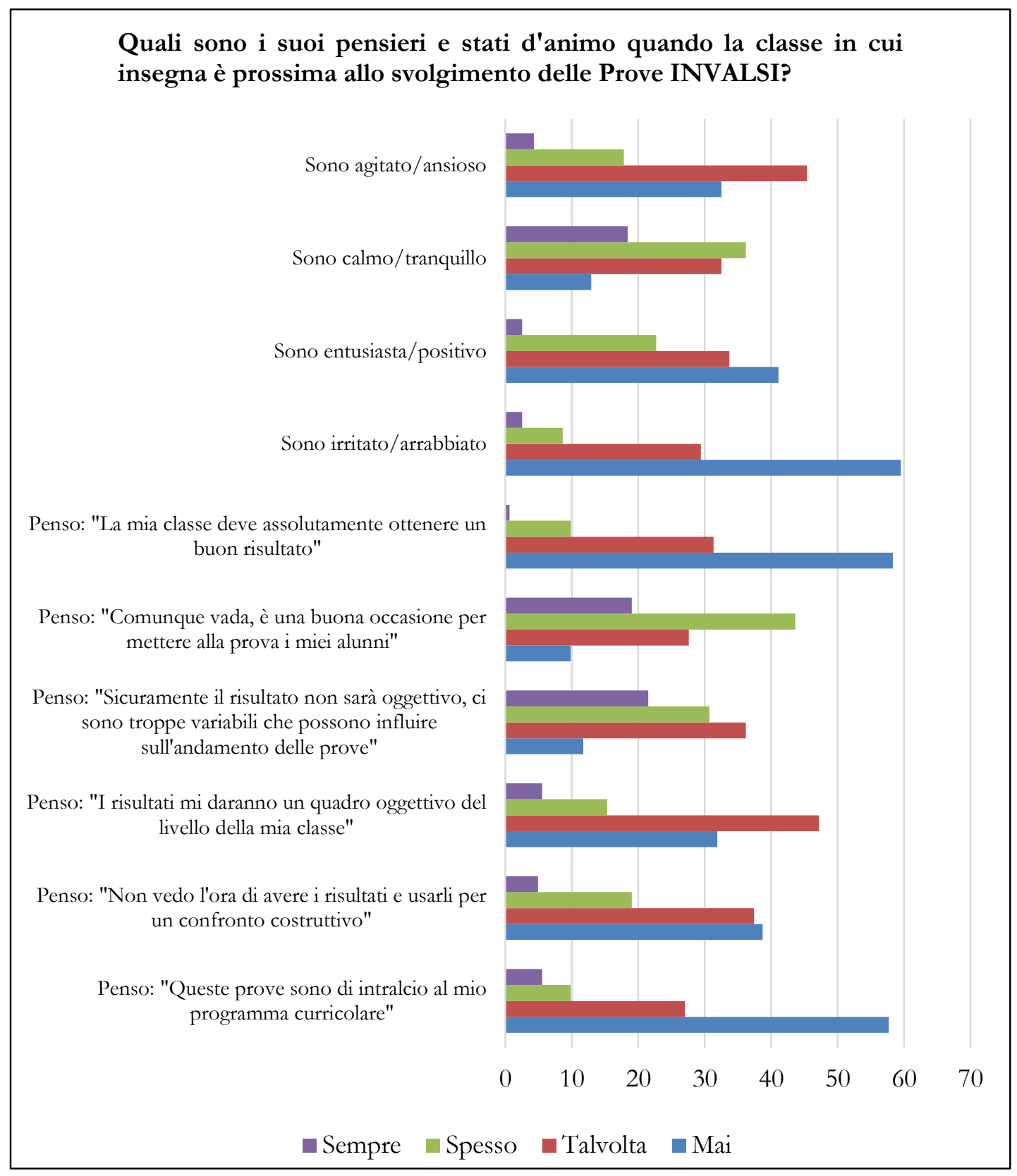

Sebbene si tratti di casi isolati, quattro insegnanti hanno espresso l'idea dell'inutilità dei test dichiarando che "non servono a nulla" (cfr. Figura15), di considerarli come un intralcio nell'attività didattica e nello svolgimento del programma predefinito, oppure definendoli come non corrispondenti ai bisogni formativi degli allievi.

La domanda numero 15 (Figura 16) si concentra sulle reazioni personali, sulle convinzioni e le aspettative individuali nei confronti delle indagini Invalsi: "Quali sono i suoi pensieri e stati d'animo quando la classe in cui insegna è prossima allo svolgimento delle prove Invalsi?". 
Nonostante il 62,58\% del campione dichiari che "Comunque vada, è una buona occasione per mettere alla prova i miei alunni", le risposte successive confutano tale impressione.

All'affermazione "Sicuramente il risultato non sarà oggettivo, ci sono troppe variabili che possono influire sull'andamento delle prove" il 52,76\% dichiara di pensarlo sempre e spesso; di conseguenza, nell'affermazione successiva "I risultati mi daranno un quadro oggettivo del livello della mia classe" il campione sceglie l'opzione talvolta nel 47,24\% dei casi e mai nel 31,90\% dei casi (Figura 15). Dalle risposte risulta come le prove Invalsi siano vissute come una possibilità offerta agli allievi, nonostante i risultati non siano avvertiti come equi e proficui nel descrivere la situazione della classe, tanto che i test Invalsi non sono ritenuti obiettivi dal 52,76\% del campione e addirittura il 31,90\% non considera mai oggettivi i risultati ottenuti.

Questi dati fanno emergere un'ulteriore questione docimologica e metodologica, soprattutto se confrontati con quelli ottenuti da "Non vedo l'ora di avere i risultati e usarli per un confronto costruttivo", da cui risulta come il 37,42\% scelga l'opzione talvolta ed il $38,65 \%$ dichiari mai (Figura 15). Sommando i due dati precedenti, si nota che il 76,07\% dei docenti del campione sostiene di non essere interessato ai risultati ottenuti per un confronto né con la propria azione didattica né con i colleghi di ambito disciplinare né con i colleghi di istituto affinché si possa delineare e realizzare un'attività di miglioramento delle proposte didattiche, in un progetto pedagogico-didattico comune e condiviso.

L'ultima opzione della domanda 15 (Figura 16) richiedeva di indicare con quale frequenza si pensa che "Queste prove sono di intralcio al mio programma curricolare". Il 57,69\% dei partecipanti al sondaggio ha dichiarato che le prove non sono mai di intralcio alle attività curricolari quotidiane, nonostante in precedenza si fosse evidenziata una distanza percepita tra le Indicazioni Nazionali del 2012, la progettazione d'istituto e curricolare e le prove Invalsi (Figure 12, 13 e 14).

La domanda numero 16 (Figura 17) si concentra su ciò che intende sondare l'indagine Invalsi nella sezione dedicata alla Riflessione linguistica. Al campione si sottopongono una serie di affermazioni a cui è necessario indicare un punteggio, da 0 a 3 , secondo una scala graduata dal minore al maggiore grado di rilevanza. Le proposte tra cui scegliere sono descritte secondo una progressiva complessità nell'attività di riflessione grammaticale. Le varie alternative si basano sugli obiettivi a cui si mira nella formulazione dei quesiti Invalsi, rimodellando le indicazioni presenti nel Quadro di Riferimento alle prove Invalsi di italiano (2018), in cui si illustrano gli scopi della sezione relativa alla Riflessione sulla lingua:

- ricorrere alla propria competenza linguistica implicita per integrare frasi e per risolvere casi, anche problematici, proposti alla riflessione;

- osservare i dati linguistici e mettere a fuoco fenomeni grammaticali anche nuovi rispetto alle consuete pratiche didattiche;

- ragionare sui dati offerti - possono essere parole, frasi, brevi testi - per confrontarli, scoprirne le relazioni, le simmetrie e le dissimmetrie, risalire alle regolarità;

- descrivere i fenomeni grammaticali;

- accedere a un approccio ai fatti di lingua (pre)scientifico piuttosto che normativo.

(Quadro di Riferimento alle prove Invalsi di italiano, 2018) 


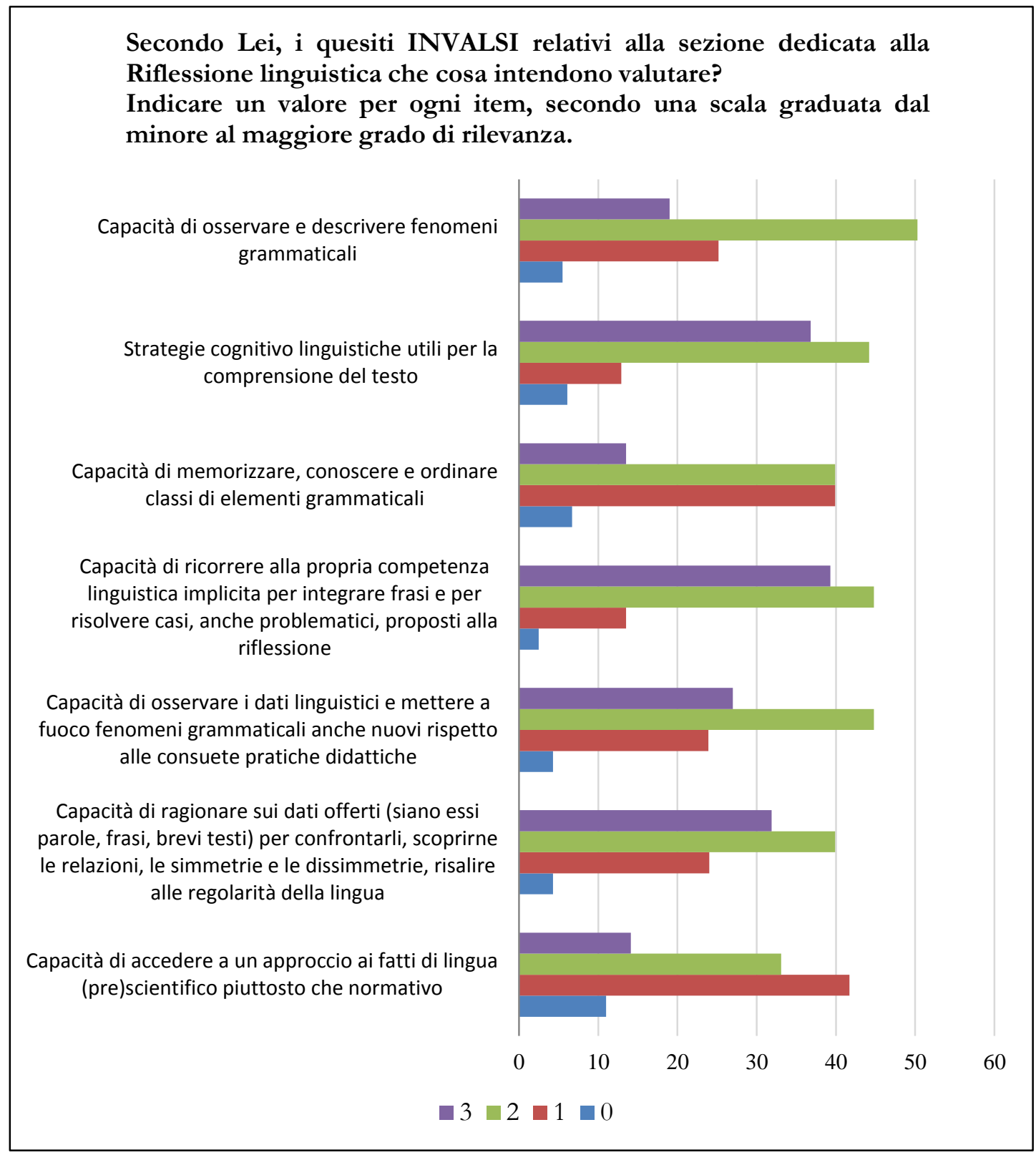

Alla "Capacità di osservare e descrivere fenomeni grammaticali": il 50,31\% del campione assegna un punteggio pari a 2; a "Strategie cognitivo linguistiche per la comprensione del testo", il 44,17\% dei docenti assegna un punteggio 2.

Sulla "Capacità di memorizzare, conoscere e ordinare classi di elementi grammaticali" il campione si divide: il 39,88\% attribuisce un punteggio 1, e una percentuale identica attribuisce punteggio 2. Sommando rispettivamente queste percentuali con quelle risultanti dalla assegnazione dei punteggi 0 e 3 , risulta che il 46,62\% dei docenti del campione crede che tale capacità non sia rilevante ed il restante $53,37 \%$ che lo sia.

Le due successive articolazioni della domanda 16 (Figura 17) riguardano due capacità insite nella competenza riflessiva; si tratta di capacità tra loro correlate nella prospettiva di un percorso di insegnamento/apprendimento della grammatica orientato a rendere gli allievi consapevoli dei processi di costruzione di significato. Alla "Capacità di ricorrere alla propria competenza linguistica implicita per integrare frasi e per risolvere casi, anche 
problematici, proposti nella riflessione", 1'84,05\% dei docenti del campione assegna un punteggio di 2 e 3 . Tuttavia, alla "Capacità di osservare i dati linguistici e mettere a fuoco fenomeni grammaticali anche nuovi rispetto alle consuete pratiche didattiche", il campione assegna un valore di 2 e 3 per il $71,78 \%$ del totale e, rispetto alla precedente capacità, il valore di 0 e 1 aumenta, passando dal 15,95\% al 28,22\% (Figura 16). Nell'educazione alla riflessione sulla lingua, la gran parte dei docenti ritiene, quindi, che si debbano tenere in considerazione le conoscenze linguistiche già possedute dagli allievi in modo implicito; nonostante ciò, si evidenzia una maggiore prudenza (cfr. le risposte con valore 1) - e in qualche caso un sostanziale disaccordo (cfr. le risposte con valore 0 ) verso la possibilità proposta dalle prove Invalsi di sondare fenomeni linguistici che esulino da un insegnamento esplicito della grammatica e dalla usuale pratica didattica.

Alla "Capacità di ragionare sui dati offerti (siano essi parole, frasi, brevi testi) per confrontarli, scoprirne le relazioni, le simmetrie e le dissimmetrie, risalire alle regolarità della lingua", il $71 \%$ del campione ha assegnato un punteggio 2 e 3 , il 29\% un punteggio 1 e 0 . Dalle risposte ottenute, sembra si possa inferire che la capacità di confrontare dati linguistici per scoprire regolarità interne alla lingua stessa, ossia una capacità sottesa ad un approccio induttivo alla grammatica, sia adottata o tenuta in considerazione da un numero relativamente alto di insegnanti del campione. Si potrà verificare se vi sia poi un'effettiva ricaduta nelle pratiche didattiche di un approccio induttivo che sviluppi questa capacità, quando si osserveranno i dati della sezione del questionario relativa alle scelte metodologico-didattiche utilizzate dagli insegnanti del campione nell'insegnamento della grammatica ( $\$ 5.2$ e sgg.).

L'enunciato "Capacità di accedere a un approccio ai fatti di lingua (pre)scientifico piuttosto che normativo" si mostra più esplicito del precedente nel fare riferimento ad un insegnamento della grammatica e ad una riflessione sulla lingua che ponga in discussione l'approccio normativo e deduttivo tradizionale. Si tratta di attivare negli allievi la capacità di ragionare sulla lingua attraverso l'esercizio di capacità cognitive come osservare, esaminare situazioni comunicative e fenomeni linguistici, registrare, ordinare e correlare dati, porsi problemi, formulare ipotesi, classificare, stabilire relazioni logiche, generalizzare e astrarre, ecc.

L'attenzione verso la capacità di osservare fenomeni linguistici con un metodo scientifico è presente sia nelle Indicažioni Naz̨ionali, come fine ultimo della riflessione sulla lingua, sia nelle prove Invalsi, come una delle capacità da sondare nelle consegne proposte.

Nelle risposte degli insegnanti del nostro campione si nota un netto aumento di coloro che attribuiscono un punteggio di 0 e 1 , ossia il 52,76\% del totale, dimostrando come più della metà degli intervistati abbia la percezione che i test Invalsi si rivelino difficilmente utili nell'esplorare le reali abilità degli allievi nel riflettere con un metodo (pre)scientifico sui fatti di lingua o che questa capacità, non essendo tra quelle prese in considerazione nella pratica didattica di questi insegnanti, non sia colta nei quesiti delle prove Invalsi ad essa dedicati.

In conclusione, la domanda 16 aveva l'obiettivo di saggiare l'opinione degli insegnanti circa le finalità dei quesiti Invalsi nella sezione dedicata alla Riflessione sulla lingua. Appare interessante notare come la maggior parte dei docenti abbia indicato il ricorso alla grammatica implicita degli allievi e la necessità di operare anche su fenomeni linguistici nuovi, rispetto a quelli presenti nella consueta attività, rispettivamente per 1'84,05\% ed il $71,78 \%$ del campione; tuttavia, emerge chiaramente, per il 53,37\% del campione, il convincimento della centralità delle conoscenze grammaticali legate alla memorizzazione, ordinamento e classificazione delle differenti classi, sebbene nel Quadro di Riferimento delle prove Invalsi del 2018 sia dichiarato esplicitamente che 
[...] nella formulazione dei quesiti di grammatica si mira, più che a misurare la capacità di memorizzare, riconoscere e denominare classi e sotto-classi di elementi, ovvero di operare una categorizzazione astratta e fine a sé stessa, a privilegiare la capacità di operare analisi di tipo funzionale e formale [...].

(Quadro di Rifermento Invalsi, 2018: 4)

Nella domanda numero 16 è stato inserito un item non contenuto nella sezione dedicata alla Riflessione sulla lingua del Quadro di Riferimento, ma presente nella sezione relativa alla comprensione testuale, in cui si intende sondare le abilità linguistico-cognitive soggiacenti all'interpretazione dei testi. La somma delle percentuali dei punteggi 2 e 3 assegnati a questo item $(80,98 \%)$, evidenzia come la maggioranza dei docenti ritenga che la dimensione grammaticale si intrecci con la comprensione (e la produzione) del testo, come illustrato all'interno del Quadro di Riferimento delle prove Invalsi di italiano, 2018:

Per dimensione grammaticale relativa alla comprensione del testo si intende la capacità di ricorrere alla cosiddetta" grammatica implicita" ed eventualmente anche alla "grammatica esplicita" per capire il testo e per risolvere dubbi di comprensione.

La dimensione grammaticale sottesa alla comprensione del testo si articola soprattutto nelle capacità di:

1. coglier egli elementi linguistici di coesione (ad esempio connettivi, catene anaforiche) e il loro apporto alla costruzione del significato del testo;

2. comprendere il significato dell'ordine "marcato" - cioè differente dalla convenzione abituale (soggetto, verbo, complementi) - delle parole nella frase;

3. riconoscere $i$ valori prosodico-intonazionali, sintattici, espressivi dell'interpunzione;

4. identificare i tempi, aspetti e modi verbali nelle loro specifiche funzioni pragmatiche e testuali (modalità, concordanza; messa in rilievo, ecc.);

5. riconoscere l'organizzazione gerarchica delle frasi complesse (ad esempio periodi costituiti da più frasi subordinate e coordinate).

(Quadro di Rifermento Invalsi, 2018: 5-6)

Ma dai dati raccolti si evidenzia anche che una parte degli insegnanti del campione attribuisce una debole attenzione agli elementi della testualità ed alla riflessione sui tipi di frase, sui connettivi e sui meccanismi anaforici (cfr. Figure 19 e 24). Ciò può far supporre che nell'ordinaria attività didattica la riflessione sulla lingua non si accompagni e non interagisca nell'esercizio della comprensione e della produzione di testi in modo organico e sistematico, come invece indicato nel Quadro Invalsi.

\subsection{Le scelte metodologico didattiche utilizzate nell'insegnamento della riflessione linguistica ed $i$ contenuti proposti nel percorso didattico}

Le ultime due domande del questionario miravano a sondare il tipo di attività di riflessione linguistica utilizzata in aula con gli allievi e i contenuti grammaticali su cui si soffermi in modo specifico la proposta didattica nella pratica quotidiana della scuola primaria.

Nella domanda 17 (Figura 18) il quesito era il seguente: "Con quale frequenza Lei svolge le seguenti attività nel fare Riflessione linguistica in classe?'. Di seguito si riporta l'ordine progressivo di frequenza emerso in base alle risposte ottenute:

1. presentazione di esempi appositamente creati ad hoc, spesso e sempre pari all' $84,05 \%$; 
(C) Italiano LinguaDue 2. 2021. M. Roccia, Le prove Invalsi e la riflessione sulla lingua nella scuola primaria: un'indagine sulla percezione degli insegnanti

2. osservazione dei fenomeni linguistici a partire dai testi narrativi letti, spesso e sempre pari al $73,62 \%$

3. osservazione dei fenomeni linguistici a partire dagli errori degli alunni nelle loro produzioni orali e/o scritte, spesso e sempre pari al 71,16\%;

4. osservazione dei fenomeni linguistici a partire dal parlato degli allievi e/o dal parlato dei nuovi media, spesso e sempre pari al 65,03\%;

5. presentazione di un "problema" linguistico che gli alunni devono risolvere tramite materiali raccolti, ipotesi e discussioni, spesso e sempre pari al 52,76\%;

6. presentazione delle regole grammaticali ed esercizi costruiti ad hoc, spesso e sempre pari all' $51,53 \%$;

7. spiegazione di regole grammaticali usando il libro di testo, seguita da esercizi applicativi, spesso e sempre pari al 38,88\%.

Figura 18. Risultati in percentuale_domanda 17

\section{Con quale frequenza Lei svolge le seguenti attività nel fare riflessione linguistica in classe?}

Presentazione della regola grammaticale ed esercizi costruiti ad hoc

Presentazione di esempi appositamente costruiti per giungere alla regola grammaticale

Spiegazione di regole grammaticali usando il libro di testo, seguita da esercizi applicativi

Osservazione dei fenomeni linguistici a partire dai testi narrativi o espositivi letti

Osservazione dei fenomeni linguistici a partire dagli errori degli alunni nelle loro produzioni orali e/o scritte

Osservazione dei fenomeni linguistici a partire dal parlato quotidiano degli allievi e/o dal parlato dei nuovi media

Presentazione di un "problema" linguistico che gli alunni devono risolvere tramite materiali raccolti, ipotesi e discussioni

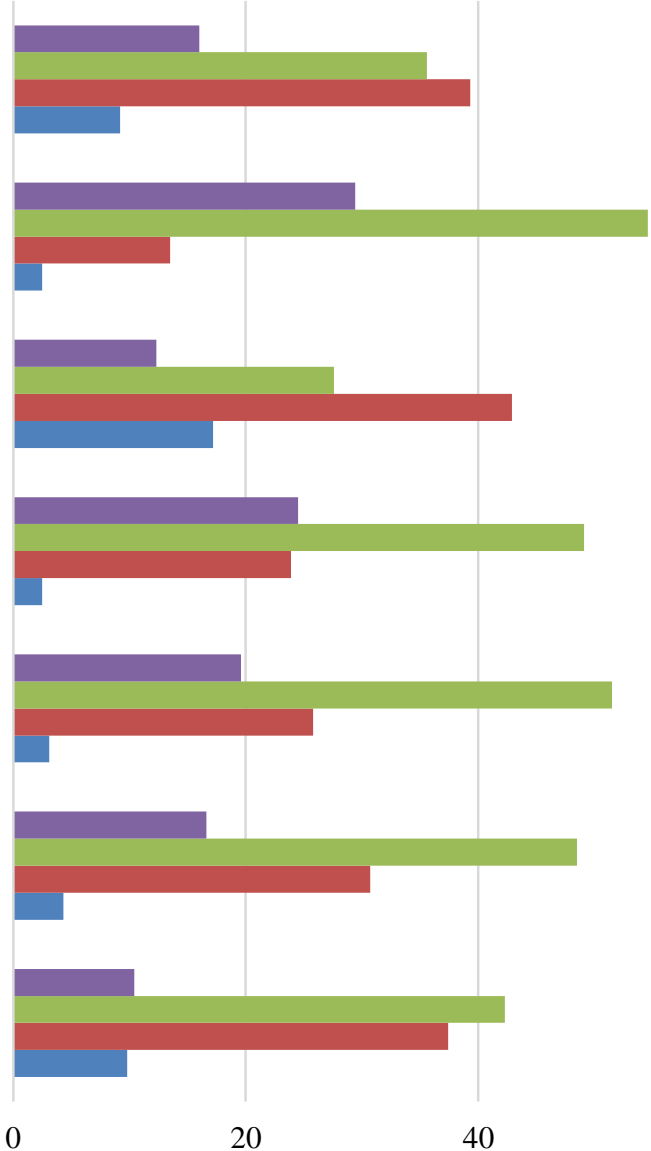

- Sempre $\square$ Spesso $\quad$ Talvolta $\quad$ Mai 
L'analisi dei dati emersi ci porterebbe a concludere che, in generale, l'approccio alla riflessione sulla lingua e la proposta didattica dei docenti della scuola primaria rispecchi ciò che si propone nelle Indicażioni Nazionali:

Nella scuola primaria la riflessione privilegia il livello lessicale-semantico e si attua a partire dai testi orali e scritti recepiti e prodotti dagli allievi.[...] La riflessione sulla lingua, se condotta in modo induttivo e senza un'introduzione troppo precoce della terminologia specifica, contribuisce a una maggiore duttilità nel capire $\mathrm{i}$ testi e riflettere e discutere sulle proprie produzioni.

(Indicarioni Nazionali 2012: 30)

\subsubsection{Gli innovatori, i tradizionalisti e gli innovatori prudenti}

Ad una rilettura più approfondita dei dati, emerge un risultato significativo che situa il campione degli insegnanti su due posizioni contrapposte, quella degli innovatori e quella tradirionalisti.

Nella domanda 17 sono indicate due attività relative a due differenti e opposte modalità di fare grammatica: procedere dalla "Presentazione della regola grammaticale ed esercizi costruiti ad hoc" oppure avviare l'attività dalla "Presentazione di un problema linguistico che gli alunni devono risolvere tramite materiali raccolti, ipotesi e discussioni". La prima alternativa è una pratica che caratterizza l'insegnamento tradizionale della grammatica, in cui l'allievo riceve le nozioni linguistiche assumendo un ruolo passivo, mentre la seconda è una procedura che segue un approccio induttivo e innovativo, in cui l'allievo ha un ruolo attivo nella co-costruzione del sapere linguistico.

In base alle risposte fornite, il campione degli insegnati si divide quasi a metà tra tradizionalisti e innovatori. Il 51,53\% dei docenti dichiara, infatti, di procedere spesso e sempre dalla regola grammaticale ed esercizi costruiti ad hoc, il 48,47\% mai oppure talvolta. Il $52,76 \%$ degli insegnanti asserisce di avviare spesso o sempre la riflessione sulla lingua dalla "Presentazione di un problema linguistico che gli alunni devono risolvere tramite materiali raccolti, ipotesi e discussioni", mentre il 47,23\% dichiara di non adottare mai o solo talvolta tale approccio didattico.

Proseguendo nell'analisi dei dati, si rileva che la maggioranza degli insegnanti: a) presenta esempi appositamente costruiti per giungere alla regola, sempre 29,45\%, spesso $54,60 \%$; b) conduce l'osservazione dei fenomeni linguistici a partire da testi narrativi o espositivi letti (sempre 24,54\%, spesso 49,08\%), quindi adottando un approccio induttivo.

Nell'esaminare i risultati del questionario, tuttavia si nota che poco più del $40 \%$ dei docenti talvolta procede attraverso "la spiegazione di regole grammaticali usando il libro di testo, seguita da esercizi applicativi", e che il 39,26\% talvolta presenta prima la regola grammaticale e poi assegna agli allievi esercizi per la sua applicazione.

Tali esiti complessivi permettono di supporre che la metodologia privilegiata per avvicinare i bambini alla riflessione linguistica sia sì quella induttiva, preferendo la possibilità di presentare esempi di lingua tratte da diverse fonti - tra cui, sempre (19,63\%), spesso $(51,53 \%)$ o talvolta $(25,77 \%)$ a partire dagli errori degli alunni nelle produzioni orali e scritte degli allievi e a partire dal parlato quotidiano degli allievi e dal parlato dei nuovi media (16,56\% sempre, 48,47\% spesso, 30,67\% talvolta) - o appositamente costruiti, per poi procedere alla ricerca ed alla descrizione della norma, tralasciando invece la presentazione della regola e la successiva applicazione della stessa in esercizi mirati.

Ciò nonostante, sembra essere adottata anche una metodologia mista, induttivodeduttiva, da parte di docenti innovatori, che alla luce di ciò potremmo definire prudenti. Infatti, la scelta ampiamente maggioritaria di presentare "esempi appositamente costruiti 
per giungere alla regola" è indice sì di un approccio induttivo (i mai sono davvero pochi, $2,46 \%$ ), tuttavia anche di una certa prudenza nel proporre una riflessione sulla lingua a partire da quella in uso dagli allievi. In questo atteggiamento che caratterizza gli innovatori prudenti emerge anche una prevalente tendenza (o abitudine ereditata da un approccio più tradizionale) ad utilizzare esempi di lingua creati per una data situazione costruita in modo artificioso, utile per concentrare l'attenzione sul fenomeno linguistico che si intende indagare, ma che, poiché inserito in un contesto comunicativo percepito come distante ed innaturale, ha poche probabilità di essere oggetto di un apprendimento significativo e duraturo.

Già nelle Dieci Tesi per un'educazione linguistica democratica del 1975, manifesto programmatico del Giscel, si indicava che, per la riflessione sulla lingua si dovesse, invece, privilegiare l'uso reale della lingua, a partire da quella degli allievi:

La sollecitazione delle capacità linguistiche deve partire dall'individuazione del retroterra linguistico-culturale personale, familiare, ambientale dell'allievo, non per fissarlo e inchiodarlo a questo retroterra, ma, al contrario, per arricchire il patrimonio linguistico dell'allievo attraverso aggiunte e ampliamenti che, per essere efficaci, devono essere studiatamente graduali.

(Dieci Tesi Giscel, tesi VIII, 3) ${ }^{14}$

Nel testo delle Dieci Tesi si chiarisce come risulti più fruttuoso l'avvio del percorso di apprendimento e di sviluppo delle capacità linguistiche degli apprendenti, compresa quella di riflettere sulla lingua, dalle loro preconoscenze linguistiche, stimolando l'osservazione e l'interpretazione dei meccanismi linguistici che già possiedono e utilizzano, a loro noti o che fanno parte della loro grammatica implicita, seppur marcati sugli assi di varietà. In tal modo si permette loro di comprenderne le caratteristiche e le deviazioni, fino a giungere alla realizzazione di forme linguistiche standard, a varietà più $\mathrm{o}$ meno formali, adeguate alle diverse situazioni comunicative, ai vari tipi di testo da produrre oralmente o per iscritto ecc., in un processo di insegnamento/apprendimento graduale.

Per le capacità sia ricettive sia produttive, sia orali sia scritte, occorre sviluppare e stimolare la capacità di passaggio dalle formulazioni più accentuatamente locali, colloquiali, immediate, informali, a quelle più generalmente usate, più meditate, riflesse e formali.

(Dieci Tesi Giscel, tesi VIII, 7)

I dati emersi con il questionario mostrano come il $71,16 \%$ del campione (Figura 18) dichiari di iniziare sempre o spesso la discussione grammaticale partendo dagli errori individuati nelle produzioni scritte ed orali degli allievi, sebbene evidenzino anche come si ricorra spesso (49,08\%, Figura 18, domanda 17) all'utilizzo di testi narrativi o espositivi letti in classe. Ciò sottolinea la presenza di una certa prudenza nell'adottare nella pratica didattica testi orali, siano essi caratterizzati da un maggiore o minore grado di formalità, nonostante tale necessità fosse già presente come elemento guida nei principi per un'educazione linguistica democratica del 1975:

Nelle capacità sia produttive sia ricettive va sviluppato l'aspetto sia orale sia scritto, stimolando il senso delle diverse esigenze di formulazione inerenti al testo scritto in rapporto all'orale, creando situazioni in cui serva passare da

${ }^{14}$ https://giscel.it/dieci-tesi-per-leducazione-linguistica-democraticaL. 
formulazioni orali a formulazioni scritte di uno stesso argomento per uno stesso pubblico e viceversa.

(Dieci Tesi Giscel, tesi VIII, 6)

Nell'esaminare le informazioni ricavate dall'indagine relative alle proposte di attività di riflessione linguistica in classe, al quarto posto si situa l'opzione "Osservazione dei fenomeni linguistici partendo dal parlato quotidiano degli allievi e/o dal parlato dei nuovi media", con una frequenza di spesso e sempre pari al 65,05\% (Figura 18), minore, rispetto alla percentuale delle risposte date ai due item precedenti.

Le possibili cause che muovono la scelta più prudente di fare più spesso ricorso ai testi scritti letti in classe (sempre e spesso 73,62\%) o a esempi costruiti ad hoc (sempre e spesso 84,05\%) per la riflessione sulla lingua, anziché ricorrere al parlato degli allievi o dei nuovi media (esigenza dichiarata di essere tenuta più o meno in considerazione: sempre il 16,56\%, spesso il 48,47\%, talvolta il 30,67\%, Figura 18), possono essere molteplici. Un primo motivo che induce gli insegnanti verso una scelta più prudente potrebbe risiedere nella cautela a distaccarsi dalla tradizione legata al testo scritto, che nella scuola primaria è tipicamente quello narrativo. Ricorrere al parlato degli allievi o dei nuovi media potrebbe quindi essere percepita come un'opzione insidiosa perché permette un minor grado di controllo sui fenomeni linguistici. Una seconda ragione è che potrebbe risultare ampia la distanza esistente tra gli esempi della lingua orale e la lingua scritta prototipica a cui si vorrebbe tendere; si potrebbe avvertire una certa lontananza tra la lingua del parlato quotidiano e la lingua obiettivo, ossia l'italiano standard, come traguardo a cui giungere.

Per quanto riguarda la lingua dei nuovi media, infine, le difficoltà che possono insorgere sono numerose: in primo luogo si tratta di un tipo di lingua in cui vi è la commistione del parlato nello scritto e viceversa, ma anche la compresenza e l'uso di diversi registri e di convenzioni linguistico-comunicative e semiotiche determinate dalle caratteristiche dei diversi media, dai loro formati, dai possibili tipi di interattività, ecc.

Le risposte date a questo specifico item del questionario mostrano che la maggioranza degli insegnanti tiene - o cerca di tenere - comunque in considerazione quanto richiamato dalle Indicażioni Narionali, nei Traguardi da raggiungere al termine della classe quinta della scuola primaria:

[L'allievo]Ascolta e comprende testi orali "diretti" o "trasmessi" dai media cogliendone il senso, le informazioni principali e lo scopo.

[...]Riflette sui testi propri e altrui per cogliere regolarità morfosintattiche $\mathrm{e}$ caratteristiche del lessico; riconosce che le diverse scelte linguistiche sono correlate alla varietà di situazioni comunicative.

È consapevole che nella comunicazione sono usate varietà diverse di lingua e lingue differenti (plurilinguismo)

(Indicażioni Nazionali per il curricolo per la scuola dellinfanzia e del primo ciclo d'istruzione, 2012: 30-31).

mentre per una parte (circa il 30\% tra mai e talvolta) tali indicazioni sembrano non essere particolarmente rilevanti.

Il raggiungimento di questi traguardi al termine della primaria risulta invece indispensabile anche alla luce delle competenze descritte nelle Raccomandarioni del Consiglio Europeo del $2018^{15}$ per quanto riguarda sia la competenza alfabetico funzionale sia quella multilinguistica: la prima consiste nella «capacità di individuare, comprendere, esprimere, creare e interpretare concetti, sentimenti, fatti e opinioni, in forma sia orale sia scritta,

${ }^{15}$ Raccomandazione del Consiglio del 22 maggio 2018 relativa alle competenze chiave per l'apprendimento permanente: https://eur-lex.europa.eu/legal-content/IT/TXT/PDF/?uri=CELEX:32018H0604(01). 
utilizzando materiali visivi, sonori e digitali attingendo a varie discipline e contesti»; la seconda «si basa sulla capacità di mediare tra diverse lingue e mezzi di comunicazione[...]».

Per quanto riguarda l'ultimo item della domanda 17 (Figura 18), nonostante le percentuali indichino come una metà gli insegnanti (gli innovatori) dichiari di procedere didatticamente nell'analisi della lingua fornendo problemi linguistici su cui riflettere (frequenza spesso e sempre al 52,72\%) e un'altra metà (i tradizionalisti) dichiari di presentare la regola grammaticale per poi procedere all'applicazione della stessa (frequenza di spesso e sempre al 51,30\%), la scelta di utilizzare il libro di testo nella spiegazione della regola grammaticale e nella sua successiva applicazione con esercizi in esso contenuti, ha una frequenza dichiarata come sempre e spesso solo del $38,80 \%$ e una frequenza di mai e talvolta del 60,12\% del campione totale (Figura18).

Questi dati suggeriscono come, nella pratica dell'insegnamento grammaticale nella scuola primaria, l'impiego del libro di testo come strumento didattico sia percepito dai docenti, sia tra gli innovatori sia tra i tradizionalisti, come poco fruibile nel realizzare il percorso di costruzione del sapere linguistico.

A questo proposito, vi è anche un'ulteriore dimostrazione di un certo disorientamento che caratterizza l'utilizzo dei testi scolastici nella pratica. Infatti, in una ricerca su un campione di docenti della scuola secondaria ed in un'analisi su un campione di libri di grammatica a tiratura nazionale in uso (Fiorentino, 2009), si evidenzia come sia presente un certo smarrimento da parte dei docenti nel loro impiego in aula e come vi siano caratteristiche comuni nelle differenti proposte editoriali ${ }^{16}$. I testi presi in esame presentano alcuni tratti comuni: i contenuti seguono un ordine tradizionale in cui si ritrovano l'ortografia, la fonologia, la morfologia(nome, articolo aggettivo, pronome, verbo, preposizione, ecc.), la sintassi(la frase semplice, la frase complessa, le subordinate, i complementi, ecc.), il lessico e la semantica; in tutte le grammatiche scolastiche analizzate si dedica ampio spazio alla morfologia ed alla sintassi; la definizione di lingua non risulta univoca; non si dà spazio alle varietà della lingua; nel definire i diversi livelli di lingua le grammatiche esaminate sembrano non seguire un modello linguistico specifico o una tassonomia organica; talvolta si riscontrano imprecisioni concettuali, fino ad una mescolanza di terminologia tradizionale ed innovativa. Rispetto alla ricerca descritta, si conclude sostenendo che

s'intuisce che gli autori dei manuali esaminati siano consapevoli dell'esigenza di rinnovare la terminologia delle categorie grammaticali, come emerge dall'introduzione di alcuni termini nuovi rispetto alla tradizione ma, al contrario, sembra che non siano convinti della necessità di innovare anche i modelli di analisi e quelli operativi, che restano invece fedeli alla tradizione (Fiorentino et al., 2009: 117).

Un'ulteriore conferma della cautela espressa nell'uso dei libri di testo da parte dei docenti della scuola primaria, si evince dalla valutazione dei libri di grammatica in uso nella scuola primaria proposta da Miglietta e Sobrero (2011). Dopo aver esaminato i libri di testo in uso nelle classi quarte e quinte della scuola primaria dagli anni Ottanta al 2010, gli autori individuano due poli entro i quali si situano i diversi testi: il primo è quello della

grammatica tradirionale, non problematica, deduttiva, basata sulla morfosintassi e sull'analisi logica tradizionale, [...] la seconda è definita come di primo

${ }^{16}$ La ricerca si è svolta nel 2008 in Campania e Molise con un questionario somministrato a un campione di docenti della Campania ed un'analisi di un campione di testi scolastici a tiratura nazionale per la scuola secondaria. 
orientamento, che comprende attività di osservazione, ragionamento induttivo, formulazione di prime ipotesi su frammenti di produzione linguistica effettiva[...] Fin qui siamo all'interno di una linea di maggiore o minore continuità con la tradizione grammaticale, che affonda le sue radici nella sistemazione aristotelica della grammatica.

Il secondo è quello della grammatica valenziale:

Ma negli ultimi 15-20 anni si è fatto strada il modello valenziale [...] Ogni libro di testo ha dunque un approccio alla didattica della grammatica che teoricamente si colloca in un posto all'interno del triangolo ideale che ha per vertici l'impostazione grammaticale tradizionale, quella di orientamento e quella valenziale (Miglietta, Sobrero, 2011: 98, 99).

«Non bisogna dimenticare, infatti, che l'editoria scolastica ha una sua specificità: deve tarare il suo prodotto non sul target dei consumatori effettivi (gli alunni), ma su quello dei decisori d'acquisto (i professori)» (Sobrero, 1997: 500). Ne consegue che a determinare l'impostazione delle grammatiche scolastiche, e quindi ad orientare le scelte degli insegnanti, è comunque l'editore scolastico, che, per esigenze di mercato (ovvero per acquisire il maggior numero di adozioni da parte dei docenti, i decisori d'acquisto) deve tarare il suo prodotto "sulle esigenze dell'insegnante "medio" o, meglio, "medio prevalente"» (Sobrero, 1997: 500). L'editore scolastico cerca quindi di "coprire tutti i segmenti di domanda, accentuando le caratteristiche di prodotto "neutro", cioè non mirato su specifici target di domanda, ovvero su scelte teoriche che limiterebbero il numero dei potenziali decisori d'acquisto. È così che, sul piano del modello grammaticale e didattico, si spiega quella che [si può definire] tecnica dell'accumulo: la grammatica descrittiva e normativa si somma al più moderno discorso delle "varietà di lingua" [...]; un pezzetto di grammatica cognitiva a un pezzetto di grammatica testuale» (Sobrero, 1997: 500) e, si può aggiungere oggi, un pezzetto di grammatica normativa ad un pezzetto (prudente) di grammatica valenziale, ecc. e, nell'accumulo, tanti esercizi e altri supporti disponibili online sulla piattaforma della casa editrice.

Come risulta dai dati raccolti attraverso il nostro questionario, si può dunque comprendere perché qualche insegnante possa non ritrovarsi in alcune scelte e proposte editoriali e optare per l'utilizzo di materiali, procedure e attività di riflessione sulla lingua alternative e più rispondenti ai bisogni linguistici dei suoi alunni. Dagli esiti del questionario si evidenzia, tuttavia, come altri insegnanti ritengano che, in ogni caso, il libro di testo sia affidabile sul piano teorico-metodologico e utile per spiegare le "regole" grammaticali ed esercitarsi con l'applicazione delle stesse, tanto da utilizzarlo spesso nel $27,61 \%$ dei casi e sempre per il $12,27 \%$, raggiungendo circa il $40 \%$ del campione totale (Figura 18).

\subsubsection{I contenuti grammaticali proposti nella scuola primaria}

Nell'ultima domanda del questionario si chiedeva ai docenti di indicare quanto spazio dedicassero ai diversi ambiti proposti nella riflessione linguistica, segnalandolo secondo una scala graduata articolata su quattro livelli: nessuno, poco, abbastanza e molto.

Gli ambiti individuati sono quelli descritti nel Quadro di Riferimento dell'Invalsi del 2018; per ognuno di essi sono stati inseriti specifici argomenti grammaticali, tratti dagli esempi presenti nel Quadro di riferimento, rispetto ai quali si richiedeva di segnalare la frequenza con cui vengono trattati in aula. 
Da un primo esame dei dati emersi (Figura 19), si desume come il numero assoluto di preferenze si concentri sulla morfologia, in particolare sulle categorie lessicali e la loro funzione nella frase. 103 dei 163 insegnanti che hanno partecipato all'indagine dichiarano di dedicare molto spazio alle categorie lessicali, raggiungendo addirittura il valore assoluto di 159 se si sommano le risposte molto ed abbastanza. Si tratta di un indicatore significativo, confermato dalle risposte fornite alla domanda della sezione sintassi, da cui si evince che lo spazio dedicato all'accordo e alle diverse categorie flessive è indicato con molto da 96 insegnanti, raggiungendo il valore assoluto di 157 se si sommano le risposte molto ed abbastanza.

Rispetto ai valori assoluti minimi, il campione dichiara di non dedicare nessuno e poco spazio alla formazione delle parole, nello specifico le polirematiche (74 sui 163 insegnanti del campione); nessuno e poco spazio viene dato alla testualità, in particolare al meccanismo dell'anafora (70 su 163 insegnanti del campione); infine, per quanto riguarda la sintassi, in particolare in riferimento ai differenti tipi di frasi, l'indicazione di nessuno e poco spazio è data da 52 docenti su 163.

Nei paragrafi che seguono, in un'interpretazione più dettagliata dei dati, si descriveranno gli esiti, espressi in percentuale, corrispondenti ai diversi ambiti di riflessione linguistica proposti nell'indagine.

Figura 19. Valori minimi e massimi espressi in valore assoluto, domanda n. 18

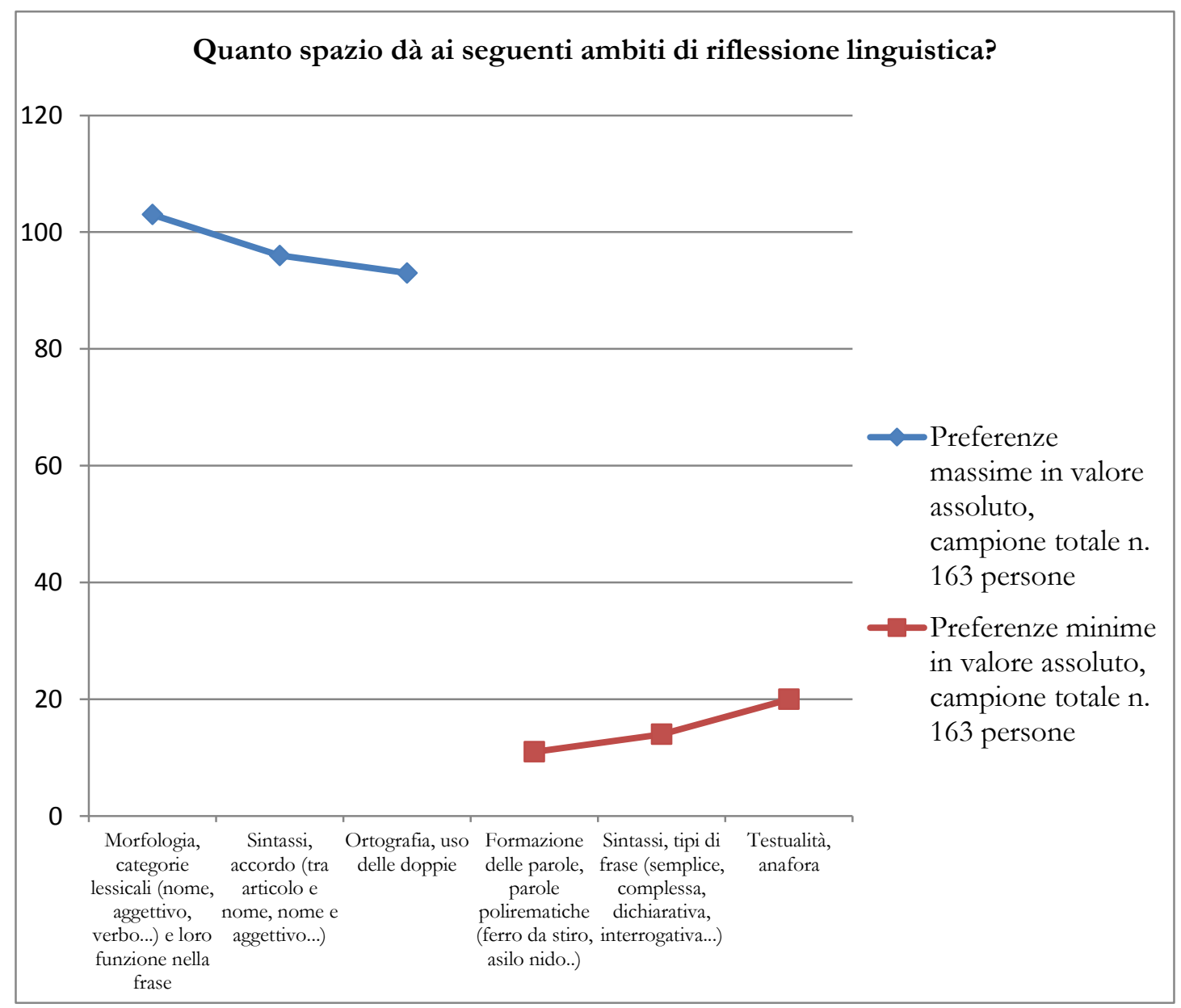


(C) Italiano LinguaDue 2. 2021. M. Roccia, Le prove Invalsi e la riflessione sulla lingua nella scuola primaria: un'indagine sulla percezione degli insegnanti

\subsubsection{I contenuti della riflessione linguistica nella scuola primaria: l'ortografia}

La prima sezione di indagine riguarda l'ortografia a cui gli insegnanti dichiarano di dedicare uno spazio molto ampio, come si può osservare nella Figura 20, in tutti gli aspetti in cui si articola la domanda 18.

Figura 20. Risultati in percentuale domanda 18_sezione 1

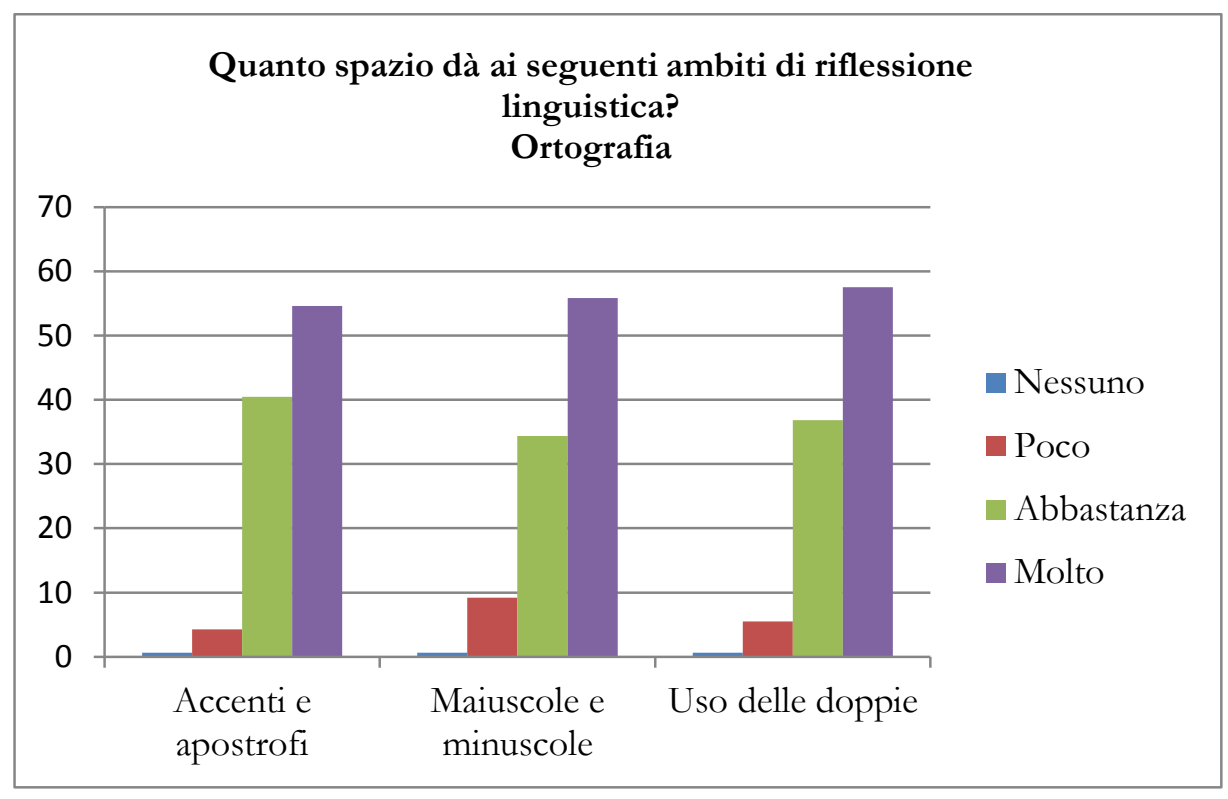

Nelle Indicazioni Nazionali, nella della sezione dedicata agli Elementi di grammatica esplicita e riflessione sugli usi della lingua (2012: 30), si raccomanda di far acquisire ed automatizzare le conoscenze ortografiche nei primi anni di scuola, in quanto si sottolinea come sia più complesso apprenderla più avanti, tuttavia se ne suggerisce un monitoraggio costante in tutti i livelli di scuola, riprendendo quanto sottolineato dalla letteratura disciplinare di riferimento (Ferreri, 1971; Lavinio, 1975; De Mauro, 1977a; Fiorentino, 2009; Serianni, 2009; Solarino, 2009; Colombo, 2011; Fornara, 2016; Lo Duca, 2018, 2021) in cui si concorda che, già nel corso della scuola primaria, i bambini giungono ad una scrittura abbastanza corretta sul piano ortografico e che le conoscenze si stabilizzano, anche negli aspetti meno regolari, durante la scuola secondaria di primo grado (Lo Duca, 2021: 7).

$\mathrm{Nel}$ Quadro di Riferimento delle prove Invalsi di italiano, all'ortografia è dedicata una sezione fin dal 2013 e, al suo interno, si ritrovano i seguenti ambiti: uso di accenti e apostrofi, maiuscole e minuscole, segmentazione delle parole (gliel'ho detto), uso delle doppie, casi di non corrispondenza tra fonemi e grafemi (uso dell'h, della $c / q$, dei digrammi, ecc.).

L'interesse verso le norme ortografiche scaturisce dal fatto che, nonostante la lingua italiana sia definita come una lingua trasparente, ossia come un sistema linguistico a cui ogni fonema corrisponde un grafema, ciò non si rivela del tutto esatto, tanto che spesso è presente una mancata corrispondenza. Sebbene la lingua italiana non sia completamente trasparente, studi comparativi hanno dimostrato che al termine della classe prima della scuola primaria, i bambini italiani, e in modo analogo i bambini con lingue similmente trasparenti come il finlandese ed il tedesco, leggono correttamente il 95\% delle parole; invece, nel caso di una lingua non trasparente come l'inglese, i bambini raggiungono una correttezza solo del 34\% (Viterbori, 2020: 223). 
Dai dati emersi dal questionario, si evince come i contenuti ortografici siano affrontati dagli insegnanti con assoluta attenzione e continuità durante l'intero ciclo della scuola primaria; ciò nonostante

sembra che, debellate nei cicli scolastici inferiori grazie al martellamento della scuola, le devianze si ripresentino nei cicli superiori. Immaginiamo che il fenomeno possa essere spiegato con un allentamento dell'attenzione e conseguentemente della riflessione esplicita. Nelle scritture più mature l'attenzione dello scrivente si focalizza per forza di cose su aspetti cognitivamente molto impegnativi (le idee da reperire, mettere in ordine, tradurre in parole adeguate e in frasi ben costruite e ben collegate, ecc.), e di conseguenza si allenta su fatti giudicati elementari, e dunque dati, a torto, come ormai assodati, tanto da essere tradotti in scrittura avendo la testa altrove (Lo Duca, 2021: 18)

\subsubsection{I contenuti della riflessione linguistica nella scuola primaria: la morfologia}

L'ambito della morfologia si attesta, oltre all'ambito ortografico, tra quelli che ottengono punteggi maggiori, quindi si dimostra come, nella scuola primaria, gli insegnanti prestino una particolare attenzione e dedichino più spazio alla trattazione degli elementi morfologici della lingua rispetto ad altri.

In particolare, gli insegnanti dichiarano di riservare molto ed abbastanza spazio alle categorie lessicali ed alla loro funzione nella frase per un totale del 97, 55\% (Figura 21). Sempre nella medesima sezione, i docenti (il 96,32\% del totale) dichiarano di dedicare molto ed abbastanza spazio alla flessione, quindi alle variabili che esprimono i diversi valori e rapporti grammaticali.

Figura 21. Risultati in percentuale domanda 18_serione 2

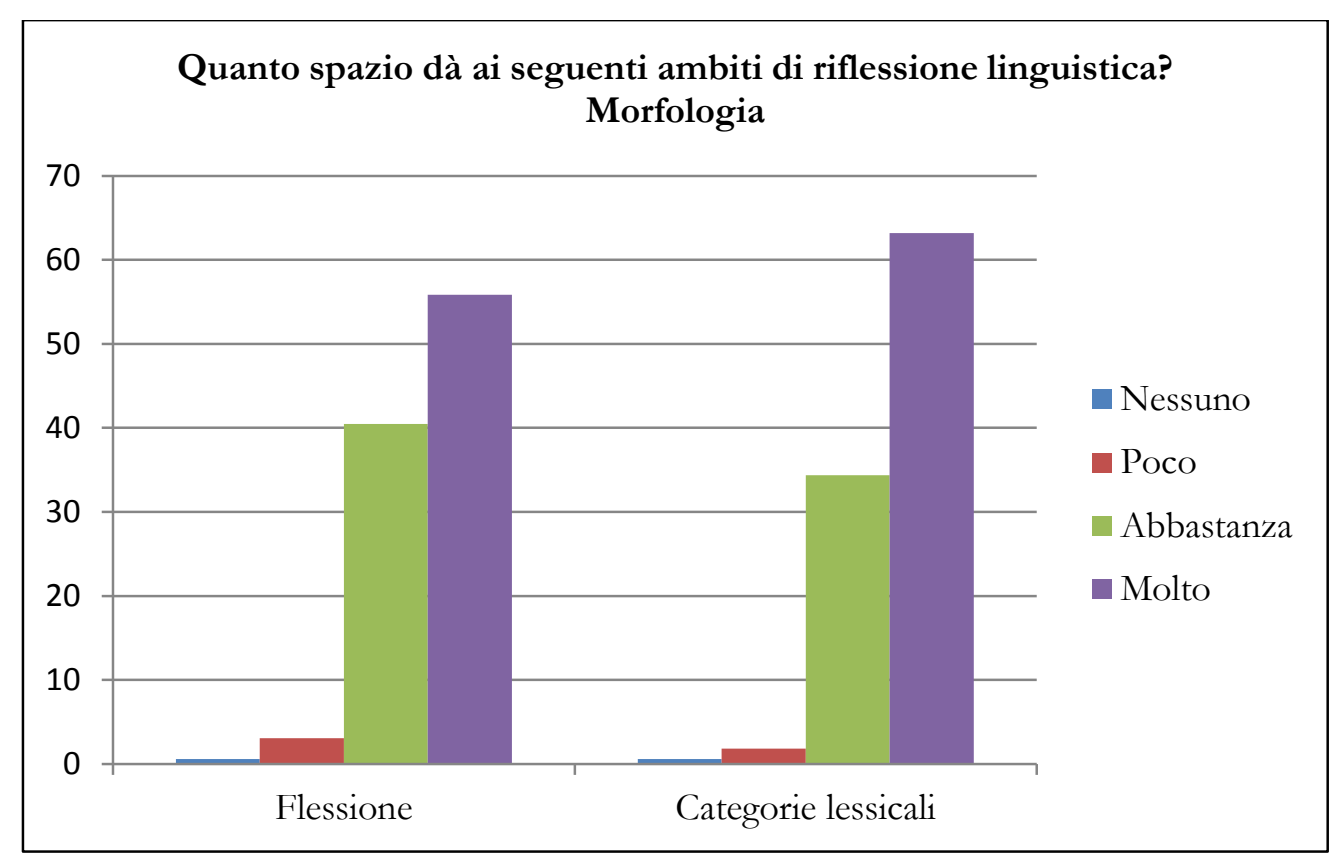

Nelle Indicazioni Nazionali le parti del discorso o categorie lessicali rappresentano uno degli oggetti della riflessione sulla lingua e della grammatica esplicita ed al termine della 
classe quinta della scuola primaria sono indicati come obiettivi a cui tendere il «riconoscere in una frase o in un testo le parti del discorso, o categorie lessicali, riconoscerne i principali tratti grammaticali» (Indicaz̨ioni Naz̧ionali, 2012: 33). Tuttavia, Lo Duca (2018: 51-53), manifesta un certo scetticismo nel lasciare così aperte le possibilità ai docenti circa le scelte da operare per la realizzazione di un sillabo progressivo ed articolato che tenga conto delle recenti proposte disciplinari e, contemporaneamente, dell'età cognitiva degli allievi. Lo Duca evidenzia ancora come, nei recenti documenti ministeriali, si ritrovino formulazioni e scansioni della disciplina riprese dai documenti precedenti, rilevando

l'assenza di una vera scansione dei contenuti. La materia grammaticale vi è richiamata in modo troppo generico, con formule che si ripetono da un segmento all'altro, e dunque il compito di scomporre in unità più piccole $\mathrm{e}$ posizionare i diversi indici nella scuola primaria viene lasciato integralmente alla decisione dei docenti (Lo Duca, 2018: 55).

Come dimostrano gli esiti emersi dall'indagine (Figura 19), nella consuetudine scolastica si sono progressivamente stabilizzate alcune pratiche: nella scuola primaria i contenuti privilegiati riguardano la morfologia e le parti del discorso, con un avvio alla sintassi della frase semplice, nella scuola secondaria di primo grado ci si concentra sulla sintassi della frase semplice e complessa e, per concludere, nel biennio della secondaria di secondo grado, si propone un ripasso della frase complessa e la trattazione, come eventuale ampliamento, di alcuni aspetti e caratteristiche della testualità (Lo Duca, 2018: 53). I dati emersi dalle risposte al questionario manifestano la scelta degli insegnanti del campione di privilegiare la sezione della morfologia dedicata alle categorie lessicali, con un assottigliamento delle percentuali dei docenti che offrono contenuti specifici sulla flessione grammaticale, ossia su quegli elementi linguistici rappresentati dai morfemi flessivi, che non interessano la categoria lessicale, ma che investono la natura grammaticale dei fenomeni linguistici osservati. Ciò nonostante, anticipando un esito legato alla sezione della sintassi (Figura 24), il campione dichiara di occuparsi della struttura della frase, concentrando i contenuti proprio sulla concordanza in un contesto sintattico definito.

\subsubsection{Il lessico}

I dati che seguiranno fanno riferimento ad item costruiti sulla base del Quadro di Riferimento dell'Invalsi (2018), Ambiti grammaticali e progressione, in cui il lessico è analizzato in due sezioni differenti: la Formazione delle parole e Lessico e semantica.

Per quanto riguarda la parte relativa alla Formazione delle parole, il Quadro prende in considerazione:

- parole semplici e parole complesse;

- parole di base e parole derivate;

- parole alterate;

- parole composte;

- parole polirematiche.

(Quadro di Riferimento dell'Invalsi, 2018: 12).

Per la parte relativa a Lessico e semantica si analizzano i seguenti aspetti:

- relazioni di significato tra parole; campi semantici e famiglie lessicali;

- polisemia;

- usi figurati e principali figure retoriche; 
(C) Italiano LinguaDue 2. 2021. M. Roccia, Le prove Invalsi e la riflessione sulla lingua nella scuola primaria: un'indagine sulla percezione degli insegnanti

- espressioni idiomatiche;

- struttura e uso del dizionario.

(Quadro di Riferimento dell'Invalsi, 2018: 12)

Nei documenti ministeriali, il lessico acquisisce un proprio status riconosciuto ed indipendente solamente nelle Indicazioni Nazionali del 2012, nella sezione denominata Acquisizione ed espansione del lessico ricettivo e produttivo.

Nelle Indicazioni Nazionali (2012) l'attenzione al lessico si ritrova in due sezioni differenti dei traguardi di competenza della classe quinta della scuola primaria: la prima riguarda l'Acquisiz̨ione e l'espansione del lessico ricettivo e produttivo e la seconda gli Elementi di grammatica esplicita e riflessione sugli usi della lingua.

Per quanto concerne l'acquisizione e l'ampliamento del lessico, gli obiettivi da raggiungere al termine della scuola primaria proposti dalle Indicarioni Narionali del 2012 sono:

- Comprendere ed utilizzare in modo appropriato il lessico di base (parole del vocabolario fondamentale e di quello ad alto uso).

- Arricchire il patrimonio lessicale attraverso attività comunicative orali, di lettura e di scrittura e attivando la conoscenza delle principali relazioni di significato tra le parole (somiglianze, differenze, appartenenza a un campo semantico).

- Comprendere che le parole hanno diverse accezioni e individuare l'accezione specifica di una parola in un testo.

- Comprendere, nei casi più semplici e frequenti, l'uso e il significato figurato delle parole.

- Comprendere e utilizzare parole e termini specifici legati alle discipline di studio.

- Utilizzare il dizionario come strumento di consultazione.

(Indicażioni Nazionali per il curricolo della scuola dellinfanzia e del primo ciclo d'Istruzione, 2012: 33)

Nella sezione Elementi di grammatica esplicita e riflessione sulla lingua le Indicazioni Nazionali (2012) riprendono e rielaborano obiettivi già presenti nelle precedenti Indicazioni risalenti al 2007. Si tratta di obiettivi da raggiungere al termine della classe quinta della scuola primaria e sono:

- Conoscere i principali meccanismi di formazione delle parole (parole semplici, derivate, composte).

- Comprendere le principali relazioni di significato tra le parole (somiglianze, differenze, appartenenza a un campo semantico)

(Indicazioni Nazionali per il curricolo della scuola dellinfanzia e del primo ciclo d'Istruzione, 2012: 33).

Il primo obiettivo, «conoscere i principali meccanismi di formazione delle parole» appartiene all'ambito della morfologia lessicale. Rappresenta, infatti, un meccanismo di innovazione linguistica che «si colloca a metà tra lessico e grammatica» (Serianni, 2006: 633) e permette di creare «parole nuove secondo modelli formativi ben determinabili» (Ibidem). Attiene, quindi, agli aspetti di regolarità della lingua, ed in particolare del lessico, studia «le parole complesse, che si sono formate tramite regole, da altre parole, con l'aggiunta di elementi formativi di varia natura» (Lo Duca, 2018: 242). Rispetto alla morfologia lessicale, si possono individuare cinque procedimenti di formazione delle 
parole: la suffissazione, l'alterazione, la prefissazione, lo sviluppo di formazioni parasintetiche e la composizione (Serianni, 2006: 634).

Il secondo obiettivo "comprendere le principali relazioni di significato tra le parole» attiene all'area semantica del lessico, quindi più vicina al significato che non alla morfologia lessicale.

Ai fini della nostra indagine, la domanda 18 è dedicata a conoscere quanto spazio sia destinato al lessico nella riflessione linguistica praticata in aula. Gli item relativi alle sezioni della domanda 18, sono stati costruiti sulla base della distinzione sul lessico presente nel Quadro di riferimento dell'Invalsi (2018: 14) in cui sono indicati due ambiti: Formazione delle parole e Lessico e Semantica e su alcuni degli obiettivi delle Indicazioni Naz̧ionali 2012.

\subsection{I contenuti della riflessione linguistica nella scuola primaria: la formazione delle parole}

Figura 22. Risultati in percentuale domanda 18_sezione 3

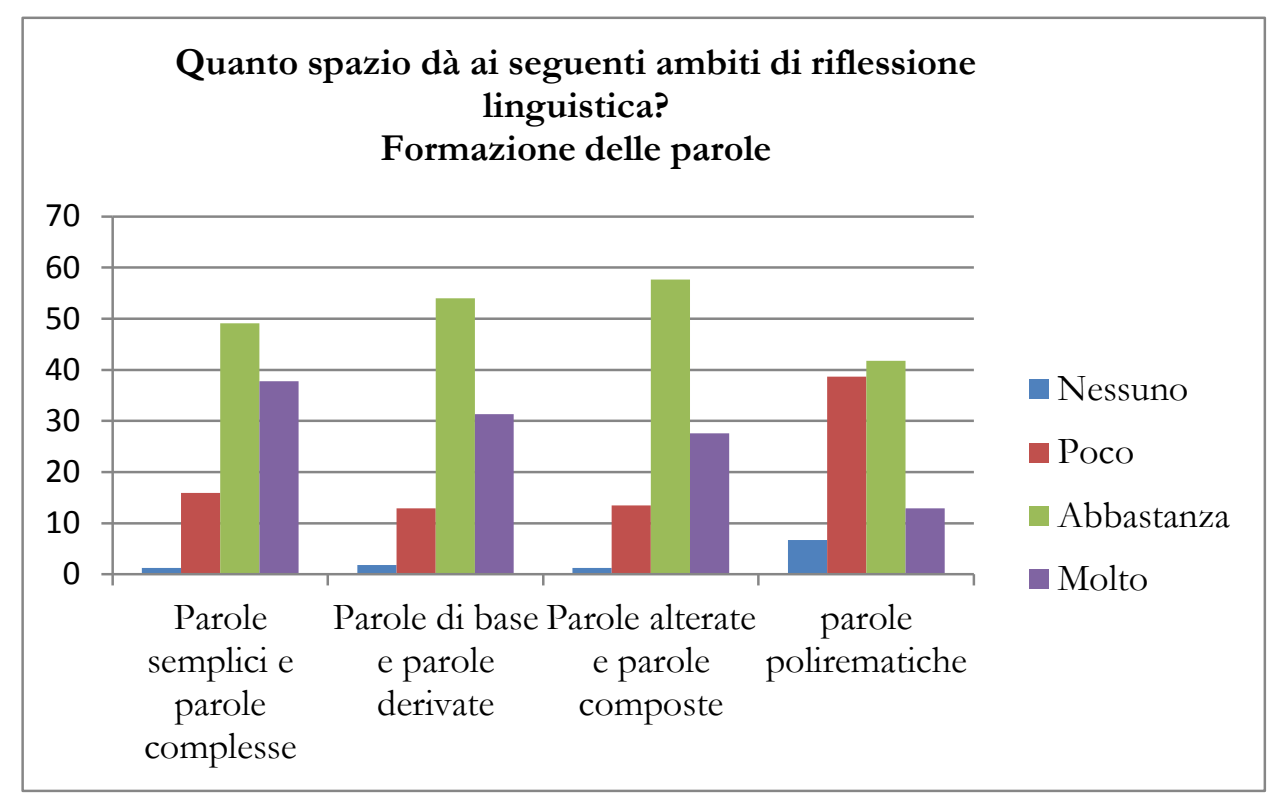

Rispetto all'ambito lessicale, dai dati si evince come i docenti manifestino di prediligere alcuni aspetti specifici. In particolare, per quanto riguarda la formazione delle parole, nella consuetudine didattica della scuola primaria, gli insegnanti del campione dichiarano di dedicare ampio spazio alle parole di base e derivate ed alle parole alterate e composte, in entrambi i casi con una percentuale che si attesta all' $85,28 \%$ nelle risposte molto e abbastanza.

Dai dati i docenti segnalano di dedicare uno spazio inferiore alla composizione delle parole, indicate come polirematiche: infatti, le percentuali abbastanza e molto si situano rispettivamente al 41,72\% ed al 12,89\% del campione (Figura 22).

\subsection{I contenuti della riflessione linguistica nella scuola primaria: il lessico e la semantica}

Per quanto riguarda il lessico e la semantica, $1{ }^{\prime} 87,11 \%$ dei docenti sostiene di dedicare molto ed abbastanza spazio alla relazione di significato tra le parole, il 77,91\% all'uso del dizionario, il 73,62\% alle parole polisemiche (Figura 23); tuttavia, rispetto all'attenzione 
offerta agli usi figurati della lingua ed alle principali figure retoriche, la percentuale si ridimensiona al $66,87 \%$ evidenziando come questi caratteri specifici della lingua letteraria non siano considerati particolarmente rilevanti o prioritari, quanto meno nell'ambito della riflessione sulla lingua.

Figura 23. Risultati in percentuale domanda 18_sezione 4

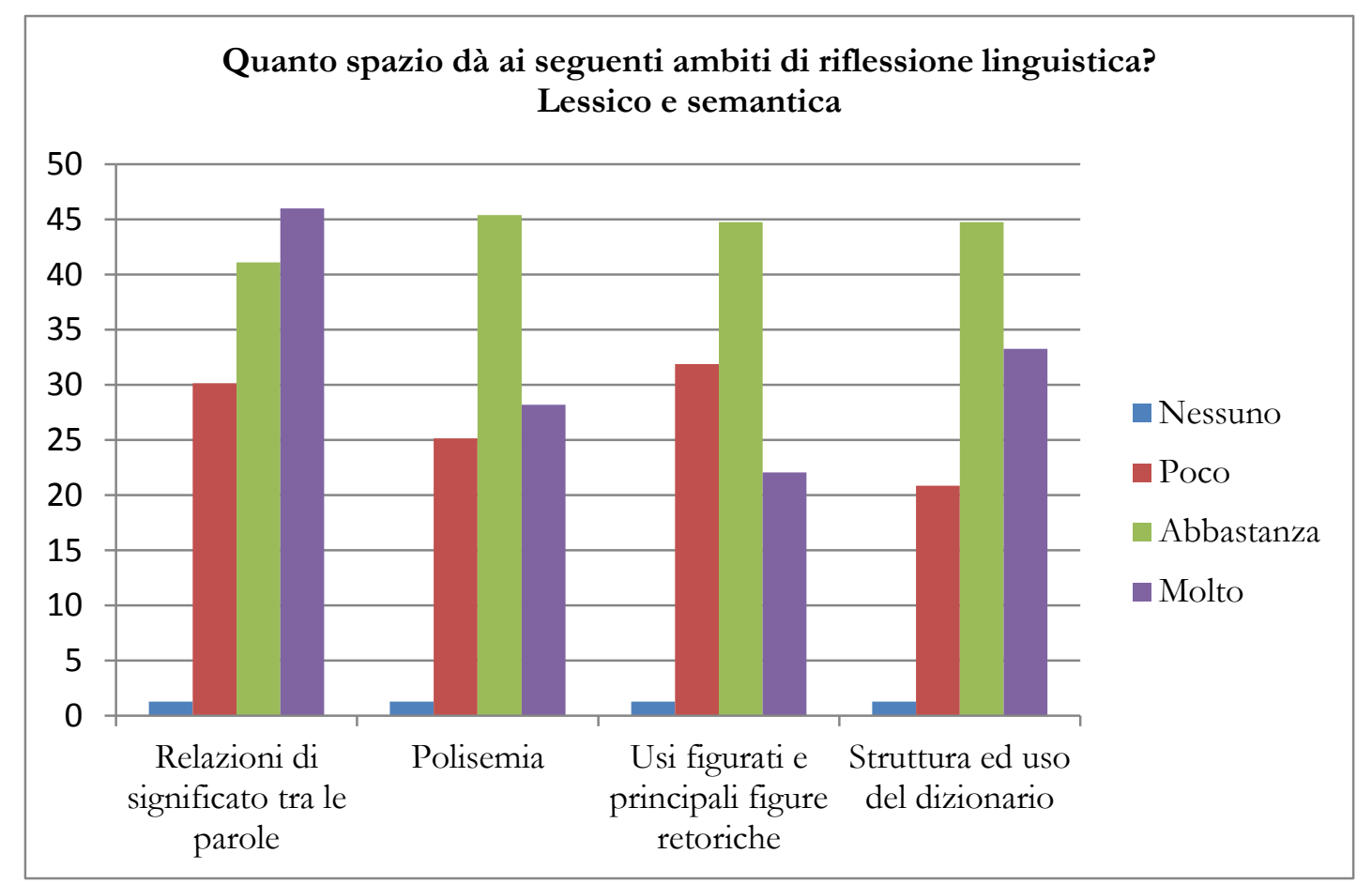

I dati emersi dal questionario dimostrano che la conoscenza delle "principali relazioni di significato tra le parole» è un obiettivo oggetto di particolare attenzione da parte degli insegnanti: infatti, la risposta molto si attesta al 46,01\% e la risposta abbastanza al 41,10\% per un totale dell' $87,11 \%$ (Figura 23). In generale, si tratta di una conoscenza che gli insegnanti si pongono come una finalità prioritaria, come documentano anche gli esiti delle prove Invalsi: nei diversi anni scolastici, i test somministrati nelle classi seconde della scuola primaria raggiungono risultati positivi tra il $60 \%$ ed il $70 \%$, in consegne in cui si richiedeva ai bambini di

collegare nomi a gruppi di aggettivi compatibili per significato (2016, C1), o di segnalare, tra coppie di parole date, quando esse «hanno lo stesso significato, cioè vogliono dire la stessa cosa o quasi la stessa cosa», e quando invece «hanno significato contrario, cioè vogliono dire due cose opposte» (2014, B1; 2015, C1) (Lo Duca, 2018: 242).

Per quanto concerne lo spazio dedicato alla «struttura e uso del dizionario» (Indicarioni Nazionali, 2012: 35), il 44,79\% degli insegnanti dichiara di offrire abbastanza spazio e il $33,13 \%$ molto spazio, per un totale del 77,92\% del campione (Figura 23). Ciò manifesta che nella pratica d'aula si ricorre frequentemente all'utilizzo del dizionario, sebbene sia necessario riprendere ciò che Colombo avvertiva già nel 2012:

«resta da stabilire se questo rifletta una pratica corrente o un rimorso per una pratica poco praticata; in ogni caso, il dizionario è concepito come lo 
strumento per la soluzione di problemi ortografici o di significato (come se non fosse noto che le definizioni dei dizionari sono più difficili del definito), mai o quasi mai come uno strumento per l'esplorazione del lessico e delle relazioni che lo strutturano» (Colombo, 2012: 13-14).

\subsubsection{I contenuti della riflessione linguistica nella scuola primaria: la sintassi}

Per quanto riguarda lo spazio dedicato alla sintassi, il 96,31\% degli insegnanti del campione, sommando le risposte molto ed abbastanza (Figura 24), dichiara di focalizzare l'attenzione soprattutto sull'accordo sintattico. Il secondo aspetto su cui converge la maggioranza delle risposte riguarda gli elementi della frase rispetto alla loro funzione logica (85,28\% di molto e abbastanza) mentre al terzo posto si colloca il tempo dedicato ai diversi tipi di frase $(68,10 \%$ di risposte molto e abbastanza).

Figura 24. Risultati in percentuale domanda 18_sezione 5

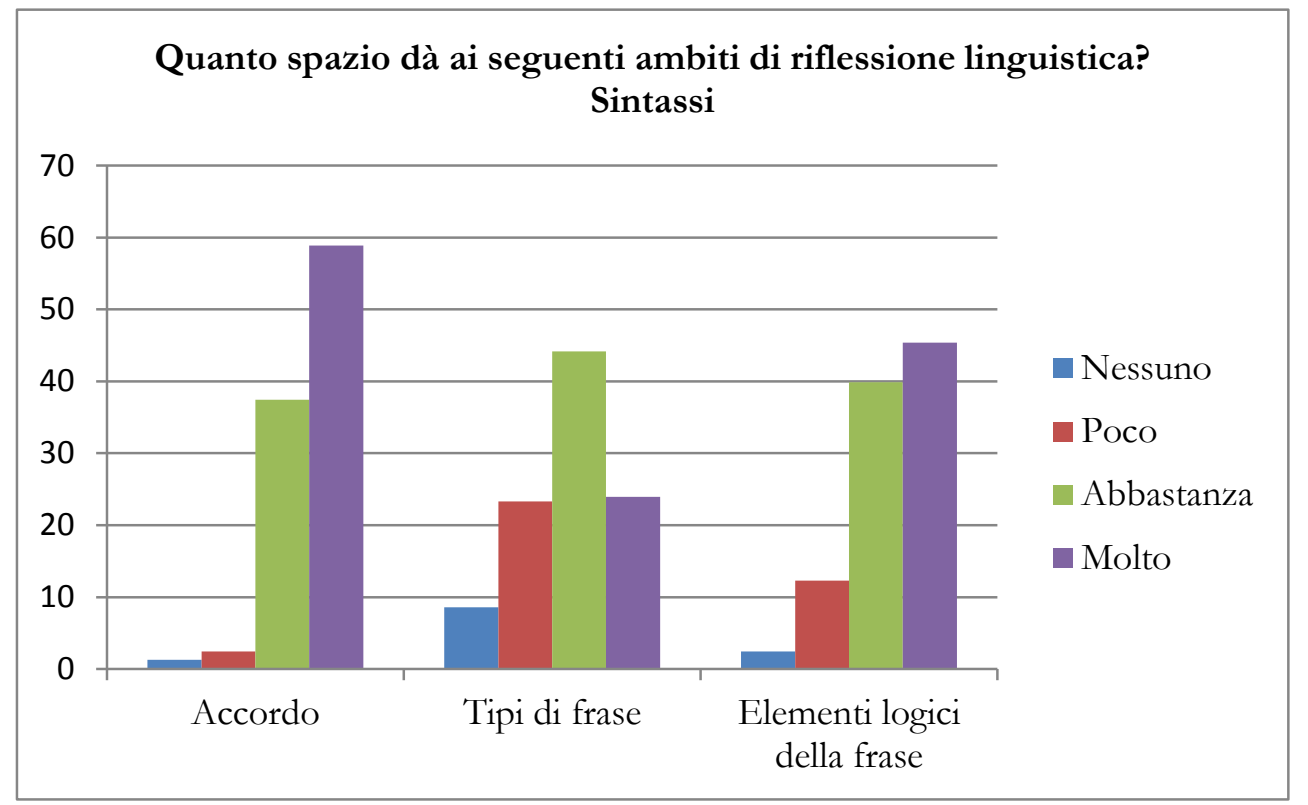

Dai dati raccolti emerge come si offra un'attenzione ampia alle funzioni logiche che esprimono le varie componenti linguistiche nel testo, mentre lo studio delle funzioni proprie della struttura della frase risultino indagate in misura inferiore.

Ciò sembrerebbe confermare la consuetudine scolastica secondo cui la riflessione sulla lingua si concentra sull'analisi grammaticale, intesa come denominazione, memorizzazione, classificazione ed individuazione delle diverse categorie lessicali e sull'analisi logica, ossia sulla denominazione, memorizzazione, classificazione ed individuazione degli elementi della frase semplice e complessa (Lo Duca, 2018: 52).

D'altronde, nell'introduzione generale dei documenti ministeriali si lascia aperta ai docenti la scelta del paradigma grammaticale da utilizzare nella pratica didattica:

Gli oggetti della riflessione sulla lingua e della grammatica esplicita sono: le strutture sintattiche delle frasi semplici e complesse (per la descrizione delle quali l'insegnante sceglierà il modello grammaticale di riferimento che gli sembra più adeguato ed efficace); le parti del discorso, o categorie lessicali; gli elementi di coesione che servono a mettere in rapporto le diverse parti della 
frase e del testo (connettivi di vario tipo, pronomi, segni di interpunzione); il lessico e la sua organizzazione; le varietà dell'italiano più diffuse.

(Indicazioni Nazionali per il curricolo della scuola dell'infanzia e del primo ciclo d'Istruizione, 2012: 30).

Tuttavia nelle Indicazioni Nazionali, invitando implicitamente gli insegnanti ad un approccio meno tradizionale alla grammatica, si suggerisce che i primi tre anni della scuola primaria siano dedicati alla riflessione sulla frase, come primo aspetto di analisi morfosintattica, ponendo come obiettivo il saper «Riconoscere se una frase è o no completa, costituita cioè dagli elementi essenziali (soggetto, verbo, complementi necessari)» (Indicazioni Nazionali per il curricolo della scuola dellinfanzia e del primo ciclo d'Istruzione, 2012: 41), mentre il riconoscimento delle classi grammaticali è un obiettivo rinviato agli ultimi due anni della scuola primaria, nonostante accada che, nella generale prassi didattica, questo rappresenti il «primo esercizio metalinguistico proposto ai bambini» (Lo Duca, 2018: 131), come per altro è confermato dagli insegnanti del nostro campione (cfr. le risposte date alla domanda 18, Figura 21).

Tra gli obiettivi che l'allievo dovrebbe raggiungere al termine della classe quinta della scuola primaria è indicato il saper «riconoscere la struttura del nucleo della frase semplice (la cosiddetta frase minima): predicato, soggetto, altri elementi richiesti dal verbo» (Ibidem, 2012: 43). Come già evidenziato da Lo Duca, negli intenti programmatici ministeriali si intende superare il modello e la scansione contenutistica tradizionale, ampliando la visione paradigmatica anche alle proposte derivanti dalla grammatica valenziale, rimandando a tale approccio con il richiamo a considerare "la struttura del nucleo della frase", il ruolo centrale del verbo e gli « altri elementi richiesti dal verbo»(Ibidem, 2012: 33). Anche nel Quadro di Riferimento per le prove Invalsi (2018: 6) vi è un implicito richiamo al modello valenziale:

Per la descrizione delle lingue si fa riferimento oggi a una pluralità di modelli teorici. Questa pluralità di proposte comporta anche la mancanza di una terminologia unitaria. Non essendo tuttavia compito dell'INVALSI indicare un modello da privilegiare rispetto ad altri, si è scelto nella formulazione delle domande di fare riferimento, in linea di massima, ai contenuti più noti e condivisi, introducendo però anche alcuni dei contenuti innovativi più assodati nel mondo della ricerca.

L'introduzione di tali contenuti innovativi si ha in numerose prove realizzate dall'Invalsi e somministrate nel corso degli anni nella scuola primaria (classe seconda: 2015, C2; 2016, B1; classe quinta: 2011, C7; 2015, C1; 2017, C3), tuttavia si può verificare quanto difficilmente questo abbia una ricaduta sull'approccio che gli insegnanti adottano riguardo alla riflessione sulla lingua, se si riosservano le risposte date alla domanda 13 punto 6 (Figura 10), punto 9 (Figura 13) e alla domanda 18 (figura 19).

\subsubsection{I contenuti della riflessione linguistica nella scuola primaria: la testualità}

Per quanto riguarda l'ultimo ambito preso in esame nel questionario, ossia la testualità, si evidenzia come il 97,55\% dei docenti dichiari di offrire molto ed abbastanza spazio alla punteggiatura, 1 ' $87,73 \%$ ai connettivi e il $57,05 \%$ al meccanismo di coesione testuale anaforico (Figura 25). 
(C) Italiano LinguaDue 2. 2021. M. Roccia, Le prove Invalsi e la riflessione sulla lingua nella scuola primaria: un'indagine sulla percezione degli insegnanti

Figura 25. Risultati in percentuale domanda 18_sezione 6

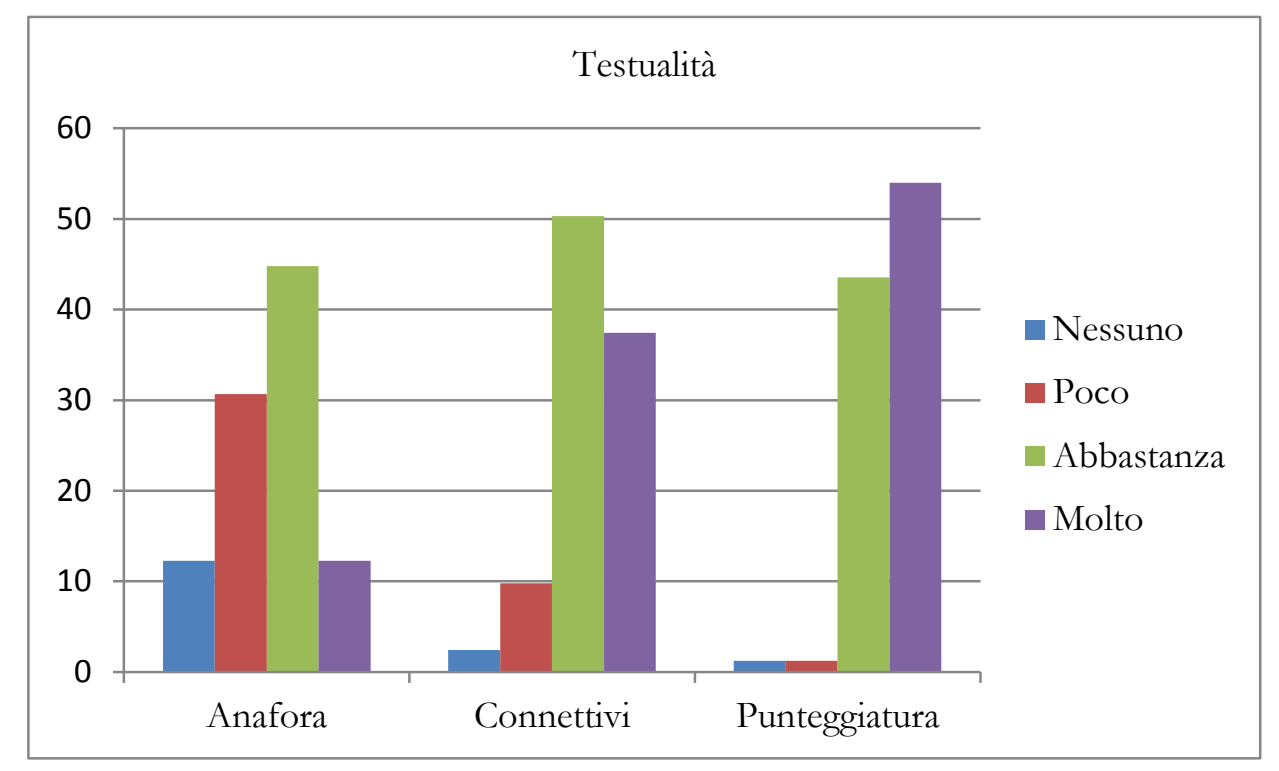

Dalle risposte ottenute alla domanda 18, sezione 2, (Figura 21) risulta come nella scuola primaria gli insegnanti del campione prestino una maggiore attenzione alla classificazione delle categorie grammaticali, con un interesse prevalente verso le funzioni logiche degli elementi nella frase anziché ai diversi tipi di frase (Figura 19), e dedichino uno spazio inferiore agli aspetti sintattici e testuali, ossia a quelle forme linguistiche sovrafrasali che permettono la comprensione e la produzione di un testo, orale o scritto, coeso e coerente. All'interno delle risposte ottenute nelle diverse sezioni della domanda 18 , in più casi si evidenzia come le categorie lessicali abbiano uno spazio più ampio nel "fare grammatica" rispetto ad altri elementi della lingua; ad esempio, nell'ambito della morfologia, l'attenzione verso queste categorie è maggiore rispetto a quella prestata ai morfemi flessivi (Figura 21). Relativamente alla sintassi, il 31,90\% del campione dichiara di dedicare poco o nessuno spazio ai diversi tipi di frase (Figura 23) e, rispondendo alla domanda 18, sezione 2 relativa alla testualità, di offrire uno spazio maggiore alla punteggiatura e minore ai connettivi e ai legami realizzati nel testo con movimenti anaforici (Figura 25).

Sebbene negli obiettivi di grammatica e riflessione sulla lingua descritti nelle Indicazioni Nazionali al termine della classe quinta della scuola primaria non siano presenti richiami particolari per l'analisi di tratti ed elementi specifici della testualità, tra gli obiettivi relativi all'abilità di lettura si pone, invece, quello di saper «[...] cogliere indizi utili a risolvere i nodi della comprensione»(Indicaz̧ioni Nazionali per il curricolo della scuola dell'infanzia e del primo ciclo d'Istruzione,2012: 42), tra i quali si collocano le relazioni anaforiche.

La debole attenzione che docenti del nostro campione dichiarano di dedicare all'anafora, ossia a quel fenomeno linguistico rispetto al quale, in campo testuale, «per interpretare un certo sintagma del testo, bisogna risalire ad un qualche elemento che compare nella porzione precedente del testo stesso, e che prende il nome di antecedente» (Lo Duca, 2018: 215), trova un riscontro negli esiti raggiunti dagli allievi nelle prove Invalsi somministrate durante i diversi anni scolastici. Da alcune analisi condotte da Toth (2016; 2017) si evince come i bambini ottengano risultati migliori nell'individuare correttamente i richiami situati in una posizione sintattica forte, ad esempio il soggetto della frase, oppure nel caso in cui i rimandi siano facilmente identificabili perché posti in una posizione centrale della narrazione $\mathrm{o}$ in un tratto significativo del racconto. Le fragilità maggiori si 
riscontrano quando gli antecedenti risultano meno salienti, concettualmente più complessi oppure indicati da riprese pronominali (cfr. Lo Duca, 2018: 218 e 219).

Per quanto riguarda la sezione della testualità dedicata ai connettivi testuali, dal questionario risulta che gli insegnanti dedicano molta attenzione nei confronti di questi legami interni al testo, coerentemente a quanto posto come obiettivo dalle Indicarioni Naz̧ionali (2012: 33) nel paragrafo Elementi di grammatica esplicita e riflessione sugli usi della lingua: saper «riconoscere le congiunzioni di uso più frequente (come e, ma, infatti, perché, quando)». Tuttavia, come illustrato da Lo Duca (2018: 224), spesso nella consuetudine didattica questa categoria è presentata come una sorta di elenco, in cui sono inseriti connettivi molto diversi tra loro sia per funzione logica sia per forma, quindi diventa difficile poter comprendere realmente ciò che possa trasformarsi in una competenza linguistica reale.

Le Indicazioni Nazionali si occupano di punteggiatura, non nella sezione dedicata alla grammatica ed alla riflessione linguistica, ma in quella dedicata all'abilità di scrittura, in cui si inserisce come uno degli obiettivi da raggiungere al termine della classe quinta della scuola primaria il saper "produrre testi sostanzialmente corretti dal punto di vista ortografico, morfosintattico, lessicale, rispettando le funzioni sintattiche dei principali segni interpuntivi» (Indicazioni Nazionali per il curricolo della scuola dell'infanzia e del primo ciclo d'Istruzione, 2012: 42).

Rispetto a quanto emerge dal questionario somministrato ai docenti, apparentemente sembra prevalere un certo interesse verso la punteggiatura (Figura 25), tuttavia si rivelerebbe utile scandagliare in modo approfondito i termini della questione: infatti, si tratta di occuparsi della punteggiatura intesa come «convenzioni in vigore», connotate da una «certa debolezza di statuto» e non scevre da un certo «disaccordo tra gli studiosi sull'interpretazione complessiva del fenomeno» (Mortara Garavelli, 2003: 5), oppure, in questo caso, di intendere la punteggiatura anche rispetto al ruolo occupato e rivestito dai segni d'interpunzione nella costruzione di legami testuali, quindi proponendo una riflessione linguistica sovrafrasale necessaria per poter ragionare su fatti di lingua e migliorare le competenze di comprensione e, in particolare, di scrittura degli allievi, magari avviando la proposta proprio dai testi prodotti dagli allievi stessi.

\section{CONCLUSIONE}

Nell'analisi dei dati emersi dal questionario, si evincono esiti utili per ulteriori approfondimenti nelle diverse aree indagate.

Per quanto riguarda l'idea di un sistema scolastico complesso, emerge con chiarezza la necessità della presenza di una Commissione Invalsi che operi attivamente, in collaborazione con lo staff del dirigente e con tutti gli organi ed i docenti dell'istituto, affinché i risultati dell'Invalsi pervenuti nelle scuole possano rivelarsi proficui, sia in una prospettiva di scambio reciproco, per realizzare una comunità educante, sia per proporre un costante dialogo pedagogico finalizzato al miglioramento didattico.

Dalle risposte alle domande del questionario relative all'organizzazione dell'istituto scolastico come sistema complesso, si ha la tacita conferma che non si realizzi una concreta interdipendenza tra l'agire e l'agito didattico, la progettazione didattica disciplinare, il curricolo d'istituto e le possibilità offerte dai risultati delle prove Invalsi ottenuti dalla singola scuola, al fine di indirizzare le proposte formative in una prospettiva di miglioramento congiunto tra gli insegnanti.

Rispetto all'attenzione che la scuola pone agli allievi con necessità specifiche, i dati relativi all'inclusione scolastica rivelano come negli istituti scolastici si attuino scelte educative improntate a rispondere ai diversi bisogni educativi degli allievi con difficoltà di 
apprendimento, ma si evince come permanga ancora la necessità di adottare, per gli allievi di madre lingua non italiana, più adeguate strategie mirate all'apprendimento dell'italiano come lingua seconda.

In merito alle finalità generali delle prove Invalsi, seppur la maggioranza degli insegnanti riconosca che esse abbiano l'obiettivo di misurare le competenze degli allievi, tuttavia emerge una certa diffidenza nei confronti dei risultati, in quanto ritenuti poco utili ai fini di un'eventuale riprogettazione dell'attività e poco affidabili nel fornire un riscontro oggettivo del livello di competenza che contraddistingue le classi esaminate, aprendo, di fatto, una questione che riguarda sia le scelte metodologiche e curricolari degli insegnanti, sia i contenuti e le modalità di valutazione e di misurazione delle competenze linguistiche da loro adottate.

Per ciò che concerne le competenze che si intendono rilevare con le prove Invalsi relative alla riflessione sulla lingua, emerge come la metà dei docenti del campione dichiari che una delle loro principali funzioni sia quella di saggiare le conoscenze grammaticali legate alla memorizzazione, all'ordinamento ed alla classificazione delle differenti categorie lessicali.

Rispetto ai contenuti affrontati nell'ambito della riflessione linguistica nella scuola primaria, i dati confermano la centralità della morfologia, in particolare l'attenzione data alle categorie grammaticali, agli accordi sintattici e agli aspetti ortografici; contemporaneamente, si segnala una debole considerazione di aspetti relativi alla formazione delle parole, agli elementi sintattici, ai diversi tipi di frase e, nell'ambito della testualità, alla funzione ed al ruolo dell'anafora nella comprensione dei testi.

Infine, i quesiti che intendevano sondare quali fossero le scelte metodologicodidattiche utilizzate nell'insegnamento della grammatica hanno permesso di individuare tre tipologie di docenti: tradiz̧ionalisti, innovatori ed innovatori prudenti. Il dato rilevante è che il campione si suddivide a metà tra tradiæionalisti ed innovatori, a dimostrazione di come sia difficile modificare, sostituire pratiche riflessive ancorate al tradizionale canone grammaticale, sebbene, da diversi decenni, tale approccio sia stato messo in discussione dalle ricerche in ambito linguistico e psico-cognitivo,e si proponga una grammatica scientificamente fondata, che faccia maturare nello studente un «atteggiamento speculativo nell'analisi della lingua» (Sabatini 1991: 19).

\section{RIFERIMENTI BIBLIOGRAFICI}

Berretta M. (1978), Linguistica ed educazione linguistica. Guida all'insegnamento dellitaliano, Einaudi, Torino.

Corrà L., Paschetto W. (a cura di) (2011), Grammatica a scuola, FrancoAngeli, Milano.

Colombo A. (2012), "Per un curricolo verticale di riflessione della lingua (parte I)", in Id. Grammatica e didattica, 4:

https: $/ /$ www.google.it $/$ url? sa $=\mathrm{t} \& \mathrm{rct}=\mathrm{j} \& \mathrm{q}=\& \mathrm{esrc}=\mathrm{s} \&$ source $=\mathrm{web} \& \mathrm{~cd}=\& v e d=2 \mathrm{a}$ hUKEwi4iszRvYn1AhVT57sIHeefABcQFnoECAMQAQ\&url=http $\% 3 \mathrm{~A} \% 2 \mathrm{~F} \%$ 2Fwww.maldura.unipd.it $\% 2 \mathrm{Fddlcs} \% 2 \mathrm{FGeD} \% 2 \mathrm{~F} 02 \mathrm{Colombo-}$

4.pdf\&usg=AOvVaw1imkveyWQJishXgf3ebZU0

Colombo A., Graffi G. (2017), Capire la grammatica. Il contributo della linguistica, Carocci, Roma.

Corda A., Marello C. (2004), Lessico. Insegnarlo, impararlo, Guerra, Perugia.

De Mauro T. (1977a), Scripta sequentur (a proposito degli "sbagli" di ortografia), in Id., Scuola e linguaggio, Editori Riuniti, Roma. 
(C) Italiano LinguaDue 2. 2021. M. Roccia, Le prove Invalsi e la riflessione sulla lingua nella scuola primaria: un'indagine sulla percezione degli insegnanti

De Mauro T. (1977b), Scuola e linguaggio. Questioni di educaz̧ione linguistica, Editori Riuniti, Roma.

De Mauro T. (2009), “A che serve la grammatica?”, in Fiorentino G. (a cura di), Perché la grammatica? La didattica dell'italiano tra scuola e università, Carocci, Roma, pp.12-22.

De Mauro T. (2011), "Due grammatiche per la scuola (e non solo)", in Corrà L., Paschetto W. (a cura di), Grammatica a scuola, FrancoAngeli, Milano, pp. 17-22.

Dieci Tesi per l'educarione linguistica democratica (1975): https://giscel.it/dieci-tesi-perleducazione-linguistica-democratica/.

Ferrari A. (2014), Linguistica del testo. Principi, fenomeni, strutture, Carocci, Roma.

Ferrari A., Zampese L. (2016), Grammatica. Parole, frasi, testi dell'italiano, Carocci, Roma.

Ferreri S. (1971), "Italiano standard, italiano regionale e dialetto in una scuola media di Palermo", in Medici M., Simone R. (a cura di), L'insegnamento dellitaliano in Italia e all'estero, Bulzoni, Roma, pp. 205-223.

Ferreri S. (2009), "Riflessività e livelli di riflessione linguistica", in Fiorentino G. (a cura di), Perché la grammatica? La didattica dell'italiano tra scuola e università, Carocci, Roma, pp. 23-32.

Fiorentino G. (a cura di) (2009), Perché la grammatica? La didattica dell'italiano tra scuola e università, Carocci, Roma.

Fiorentino G., Cacchione A., De Simone G., Di Vizio A. (2009), "La grammatica a scuola: prassi didattica, strumenti di lavoro e acquisizione di conoscenze", in Fiorentino G. (a cura di), Perché la grammatica? La didattica dell'italiano tra scuola e università, Carocci, Roma, pp.109-124.

Fornara S. (2010), La punteggiatura, Carocci, Roma.

Fornara S. (2012), Alla scoperta della punteggiatura. Proposte didattiche per riflettere sul testo. Carocci, Roma.

Lavinio C. (1975), L'insegnamento dellitaliano. Un'inchiesta campione in una scuola meda sarda, Edes, Cagliari.

Laudanna A., Voghera M. (2011), “Apprendimento e insegnamento implicito e esplicito della grammatica" in Corrà L., Paschetto W. (a cura di), Grammatica a scuola, FrancoAngeli, Milano, pp. 23-35.

Lo Duca M. G. (1997), Esperimenti grammaticali. Riflessioni e proposte sull'insegnamento della grammatica dell'italiano, Carocci, Roma.

Lo Duca M. G. (2018), Viaggio nella grammatica. Esplorazioni e percorsi per i bambini della scuola primaria, Carocci, Roma.

Lo Duca M. G. (2019), "Riflessione sulla lingua e modello valenziale: cominciamo dalla primaria?, in Italiano LinguaDue, 11, 2, pp. 255-265:

https://riviste.unimi.it/index.php/promoitals/article/view/12774.

Lo Duca M.G., Toth Z. (2021), "La competenza ortografica nelle prove INVALSI", in Italiano a scuola, 3, pp. 1-38: https://italianoascuola.unibo.it/article/view/12931.

Miglietta A., Sobrero A. A. (2011), "Pratiche di grammatica nella scuola elementare: un'indagine", in Corrà L., Paschetto W., Grammatica a scuola, FrancoAngeli, Milano, pp. 97-112.

Ministero dell'Istruzione dell'Università e della Ricerca (2012), Indicazioni nażonali per la costruzione del curricolo della scuola dell'infanzia e del primo ciclo d'istruzione: http://www.indicazioninazionali.it/wpcontent/uploads/2018/08/Indicazioni_Annali_Definitivo.pdf.

Mortara Garavelli B. (2003), Prontuario di punteggiatura, Laterza, Roma-Bari.

Quadro di Riferimento di italiano della prova Invalsi (2018):

https://invalsi-areaprove.cineca.it/docs/file/QdR_ITALIANO.pdf. 
(C) Italiano LinguaDue 2. 2021. M. Roccia, Le prove Invalsi e la riflessione sulla lingua nella scuola primaria: un'indagine sulla percezione degli insegnanti

Sabatini F. (1991), "La riflessione sulla lingua in un'ipotesi di curricolo complessivo", Marello C., Mondelli G. (a cura di), Riflettere sulla lingua, La Nuova Italia, Firenze, pp. 15-24.

Serianni L. (2006), Grammatica italiana. Italiano comune e lingua letteraria, con la collaborazione di Castelvecchi A., UTET, Torino.

Serianni L. (2011), "Dal testo di grammatica alla grammatica in atto", in Corrà L., Paschetto W. (a cura di), Grammatica a scuola, FrancoAngeli, Milano, pp. 73-96.

Serianni L., Benedetti G. (2015), Scritti sui banchi. L'italiano a scuola tra alunni e insegnanti, Carocci, Roma.

Solarino R. (2009), Imparare dagli errori, Tecnodid, Napoli.

Sobrero A. A. (1997), "Il peso della grammatica", in Calò R., Ferreri S. (a cura di), Il testo fa scuola. Libri di testo, linguaggi ed educarione linguistica, Quaderni del Giscel, La Nuova Italia, Firenze, pp. 489-502.

Toth Z. (2016), "Riconoscimento delle relazioni anaforiche nelle prove INVALSI (20102014)", in Lingue antiche e Moderne, 5, pp. 227-247:

https://www.researchgate.net/publication/311674685_Riconoscimento_delle_rel azioni_anaforiche_nelle_prove_Invalsi_2010-2014.

Toth Z. (2017), "L'anafora nelle prove INVALSI", in Vedovelli M. (a cura di), L'italiano dei nuovi italiani, Aracne, Roma, pp. 329-342.

Viterbori P. (2020), "La lettura e la comprensione del testo scritto", in Amoretti G., Morra S., Usai M. C., Viterbori P. (a cura di), Processi cognitivi e apprendimento scolastico, Carocci, Roma, pp. 223-242. 